\title{
Articoli \\ Classificazione, diagnosi ed ICD-10. I - Principi generali e considerazioni critiche *
}

\author{
GIOVANNI de GIROLAMO \\ Division of Mental Health, World Health Organization, Geneva
}

\begin{abstract}
Riassunto. Scopo - Discutere i principali problemi teorico-pratici connessi alla classificazione in campo medico e psichiatrico, e presentare la struttura generale della decima revisione della Classificazione Internazionale delle Malattie (ICD-10), entrata ufficialmente in vigore dal gennaio del 1993. Risultati - Sono dapprima discussi i fondamenti epistemologici della classificazione, le varie strategie classificatorie adottate in campo medico e psichiatrico ed i principali modelli classificatori esistenti. Sono quindi analizzati i problemi relativi alla attendibilità ed alla validità delle diagnosi psichiatriche, e vengono descritte le strategie che possono essere impiegate per migliorarle. Infine sono discussi i principi che hanno informato ia stesura dell' ICD-10 ed i field trials effettuati al fine di preparare il testo finale delle direttive diagnostiche. Conclusioni - La classificazione in psichiatria rappresenta un passaggio obbligato e necessario per rendere possibile la sistematizzazione delle nuove conoscenze e la comunicazione tra operatori, ricercatori ed utenti. La decima revisione dell' ICD rappresenta lo sforzo più ampio sinora compiuto in campo psichiatrico al fine di predisporre un sistema classificatorio rigoroso che, nel contempo, sintetizzi approcci e culture psichiatriche differenziate.
\end{abstract}

Parole chiave: diagnosi, classificazione, ICD-10.

Summary. Objective - To discuss some theoretical and practical problems related to classification in the medical and psychiatric fields, and to present the general structure of the Tenth Revision of the International Classification of Diseases (ICD-10), officially adopted as from January 1993. Results - This paper discusses the epistemological basis of classification, the various classification strategies adopted in the medical and psychiatric fields and the main theoretical models employed in this area. The problems related to the reliability and validity of psychiatric diagnoses are then reviewed, and the different strategies to be used in order to improve them are presented. Finally the principles which have led the development of the ICD-10, and the field trials carried out in order to test the appropriatness of this system are discussed. Conclusions - Ir the psychiatric field classification is essential in order to make possible an ordered systematization of new knowledge and to facilitate the communication between health workers and users of services. The ICD-10 represents the biggest effort ever carried out at an international level to build a reliable classification incorporating different psychiatric traditions.

Key words: diagnosis, classification, ICD-10.

Ricevuto il 4.3.1993 - Revisione ricevuta il 10.5.1993 - Accettato il 17.5.1993.

* Le opinioni espresse in questo articolo sono sola responasbilità dell'autore.

Indirizzo per la corrispondenza: Dr. G. de Girolamo, Division of Mental Health, WHO, 1211 Geneva 27, Switzerland.

Fax. (+ 41) 22-791.0746 


\section{INTRODUZIONE}

Dal gennaio del 1993 è ufficialmente entrata in vigore, a livello internazionale, la $10^{\mathrm{a}}$ revisione della Classificazione Internazionale delle Malattie (ICD-10), curata dalla World Health Organization. Il capitolo $\mathrm{V}$ dell'ICD-10 è quello relativo alle sindromi ed ai disturbi psichici e comportamentali (1992), e la sua introduzione rappresenta senza dubbio una tappa significativa per la clinica e la ricerca in psichiatria, oltreché il risultato di un largo sforzo collaborativo intrapreso a livello internazionale. Scopo di questo contributo è innanzitutto quello di discutere alcuni concetti fondamentali relativi alla classificazione ed alla tassonomia in campo psichiatrico; si analizzeranno i problemi connessi all'approccio diagnostico-nosografico in psichiatria e le questioni relative alla attendibilità ed alla validità delle diagnosi psichiatriche, ed alle conseguenze che i mutamenti che intervengono in campo diagnostico hanno sui risultati della ricerca epidemiologica. Infine si descriverà la struttura fondamentale dell'ICD-10.

\section{LA CLASSIFICAZIONE COME FONDAMENTA- LE ATTIVITÀ COGNITIVA}

\section{Principi e funzioni della classificazione}

La classificazione è un processo cognitivo fondamentale nell'operare scientifico così come nella vita quotidiana, giacché consente di strutturare ed ordinare il mondo fenomenologico in base a caratteri e lineamenti simili o differenti tra le persone o gli oggetti, ed alla relazione che intercorre tra essi. Il termine classificazione si riferisce pertanto «all'attività volta ad ordinare o arrangiare gli oggetti in gruppi o classi sulla base delle loro relazioni» (Sokal, 1974): in altre parole la classificazione è un processo volto alla creazione di categorie. In campo scientifico, la classificazione occupa un ruolo preminente sia nella fase iniziale che in quella più avanzata del procedimento scientifico, ed ha il fine precipuo di ordinare in qualche modo la pluralità delle osservazioni e dei dati raccolti e di discernere quelli rilevanti da quelli secondari (Federspil \& Scandellari, 1986). Inoltre, mentre nelle fasi iniziali della ricerca scientifica una classificazione adempie a finalità eminentemente pratiche, con il progressivo affinarsi dell'elaborazione concettuale essa viene ad assumere un valore più propriamente teorico e diviene a sua volta generatrice di ipotesi da mettere alla prova. Va inoltre sottolineato che «il momento classificatorio non costituisce mai un puro prodotto dell'osservazione, ma, dipendendo sempre la classificazione dall'assunzione (più o meno esplicita) di uno specifico punto di vista, rappresenta essa stessa un tipo di ipotesi» (Federspil \& Scandellari, 1986).

In campo medico, come ha sottolineato Feinstein (1972), un sistema classificatorio adempie a 3 funzioni precipue:

1. una funzione di denominazione, attraverso la quale viene dato un nome comune ad un gruppo di fenomeni; pertanto una classificazione rende possibile e favorisce la comunicazione tra clinici, ricercatori ed utenti;

2. una funzione di qualificazione, volta ad arricchire il contenuto informativo del nome (o della categoria diagnostica) aggiungendovi delle importanti caratteristiche descrittive quali i sintomi tipici propri del disturbo in questione, l'età di insorgenza, la sua gravità, ecc.;

3. una funzione di predizione, che ha lo scopo di fornire un giudizio di natura probabilistica circa il decorso e l'esito del disturbo stesso, così come un giudizio circa la risposta al trattamento; questa funzione dovrebbe essere considerata particolarmente importante in quanto, in ultima analisi, dovrebbe rappresentare una validazione dell'ipotesi diagnostica stessa.

Sono fondamentalmente due le attività cognitive coinvolte nelle procedure di classificazione medica: la prima è tassonomica, e consiste nella demarcazione, definizione e messa a punto delle categorie che saranno impiegate per la diagnosi: ad esempio la categoria "schizofrenia paranoide". Il termine "tassonomia" è tuttavia anche impiegato per riferirsi alla metateoria ed allo studio sistematico del processo classificatorio di per sè: in questo ambito esso abbraccia la logica, i principi ed i metodi impiegati per costruire sistemi classificatori, così come le regole attraverso cui sono attuate le identificazioni a fini classificatori (Millon, 1991). La seconda attività è invece diagnostica: essa consiste nel fornire regole per l'identificazione di uno specifico disturbo in una determinata persona, rendendo possibile la selezione di una (o più) categoria/e relativa/e alla persona considerata.

In medicina, parallelamente allo sviluppo delle conoscenze, si è assistito al graduale passaggio da modelli di classificazione di tipo sintomatologico, a modelli classificatori centrati sulla identificazione 
della sede e della natura dei fenomeni morbosi (grazie soprattutto allo sviluppo dell'anatomia patologica), sino quindi all'adozione di modelli di tipo più strettamente eziologico, basati sull'identificazione degli agenti causali (Federspil, in stampa; Federspil \& Scandellari, 1986; Scadding, 1988). Attualmente la nosografia medica si presenta estremamente disomogenea ed ispirata a criteri eclettici: pertanto alcune malattie sono classificate secondo un criterio di tipo sintomatologico-sindromico (come nel caso di larga parte dei disturbi psichiatrici), altre sono denominate e classificate a livello morfologico (ad esempio: in farto del miocardio, peritonite), altre rappresentano entità classificate a livello di una accertata disfunzione biochimica (ad esempio: porfiria), altre ancora costituiscono anormalità funzionali e sono classificate come tali (ad esempio: fibrillazione atriale), ed infine alcune prendono il proprio nome e la propria valenza classificatoria dal nome dell'agente microbico coinvolto (ad esempio: amebiasi) (Feinstein, 1977). Da ciò ne discende che, essendo le malattie, negli attuali sistemi nosografici, definite secondo criteri di tipo differente, esse non possono essere ordinate gerarchicamente (Scadding, 1993).

È possibile definire le caratteristiche distintive di una classificazione ideale in campo medico (e psichiatrico)? Essa dovrebbe rispecchiare quanto più possibile l'ordinamento presente in natura, dovrebbe essere costruita secondo le più rigorose regole metodologiche e dovrebbe essere testabile, parsimoniosa e non-tautologica (Parshall \& Priest, 1993).

Per quanto attiene alla classificazione dei disturbi mentali, come ha sottolineato Kendell (1984), essa dovrebbe essere basata, in maniera mutuamente esclusiva, su una delle seguenti dimensioni: (a) sintomatologia; (b) prognosi, o (c) eziologia. L' ultimo tipo di classificazione appare euristicamente più feconda, più precisa e più attendibile, comporta i massimi vantaggi dal punto di vista operativo (soprattutto per le possibili implicazioni terapeutiche), ed è inoltre quella verso cui, come si è detto, si è gradualmente orientata l'intera nosografia medica. In campo psichiatrico, però, la limitatezza delle conoscenze disponibili a livello ezio-patogenetico rende una classificazione siffatta al momento impraticabile, e limitata a quei (pochi) disturbi ad eziologia dimostratamente organica. Inoltre, vi sono tre importanti limiti connessi all'adozione di un sistema classificatorio psichiatrico (e non solo psichiatrico) di tipo eziologico (Rutter \& Gould, 1985): innanzitutto la maggior parte dei disturbi psichiatrici hanno una genesi multifattoriale, cosicché una loro classificazione basata sull'identificazione di un solo fattore eziologico finirebbe con il rappresentare, in molti casi, una ipersemplificazione (anche se appare comunque possibile riconoscere ed utilizzare a fini classificatori un movente eziologico ritenuto fondamentale); in secondo luogo una singola causa di malattia può condurre a quadri clinici ed a esiti molto diversi tra loro: basti pensare, come chiaro esempio di cio, al diabete o alla tubercolosi; e tale obiezione appare di particolare rilievo in psichiatria, ove l'eterogeneità dei decorsi e degli esiti è evenienza comune. Infine va notato che il trattamento e la prognosi possono dipendere dalla natura del disturbo piuttosto che dalla sua eziologia: ad esempio, il fumo può essere all'origine del tumore del polmone, del catarro bronchiale o di alterazioni della capacità polmonare, ma le conseguenze di ciascuno di questi quadri morbosi, sia in termini di mortalità che di morbidità, sono marcatamente differenti nonostante il movente eziologico comune.

Una classificazione basata sulla prognosi, pur essendo stata talora utilizzata in psichiatria, a partire dalla nozione di demenza precoce introdotta da Kraepelin, è resa problematica dal fatto che, a rigor di termini, attraverso essa si renderebbe possibile un giudizio diagnostico solo retrospettivamente, quando cioè il corso dell'evoluzione di un disturbo è chiaramente determinato; pertanto siffatta classificazione impedirebbe l'adozione tempestiva di un qualsivoglia intervento terapeutico. Inoltre, l'eterogeneità dei decorsi e degli esiti che caratterizza gran parte dei disturbi e delle sindromi psichiatriche, già notata prima, rende tale classificazione poco affidabile.

A causa, quindi, della limitatezza delle conoscenze ezio-patogenetiche disponibili e dei limiti operativi e concettuali connessi ad una classificazione centrata sulla prognosi, una classificazione eminentemente basata sui sintomi, e sulle loro costellazioni, si è imposta come la più agevole e praticabile (Kendell, 1974): in tal senso «le caratteristiche che definiscono la maggior parte delle affezioni psichiatriche non sono altro che la sindrome clinica loro peculiare»" (Kendell, 1984). Una sindrome è rappresentata da un insieme di segni e sintomi che tendono a manifestarsi insieme e che sono caratterizzati da un decorso temporale più $o$ meno caratteristico e della quale non è nota la causa: è evidentemente implicita in siffatta modellizzazione classificatoria la speranza che il progresso delle conoscenze renda possibile l'elucidazione dei meccanismi causali sottostanti alle differenti sindromi ed alla covariazione dei segni, dei sintomi, del decorso, della prognosi e della risposta al trattamento che le caratterizza (o le dovrebbe caratterizza- 
re): da questo punto di vista «La covariazione costituisce l'essenza della scienza descrittiva e la pietra di volta del pensiero scientifico» (Meehl, 1985). Le categorie diagnostiche così ottenute sono pertanto essenzialmente fondate su considerazioni attuariali (Cooper \& Morgan, 1981), poiché osservazioni compiute su ampi numeri di pazienti vengono messe insieme per giungere alla definizione di tali categorie. L' importanza della numerosità delle osservazioni per l'intera nosografia medica era stata chiaramente notata da Foucault (1969), che aveva osservato come «La conoscenza medica non avrà certezza che proporzionalmente al numero di casi considerati dal suo esame $[. .$.$] La certezza medica si costituisce non a$ partire dalla individualità completamente osservata, ma da una molteplicità interamente percorsa da fatti individualirs.

Così come si possono ipotizzare modelli classificatori basati su dimensioni diverse, è parimenti possibile individuare diversi tipi di diagnosi (Cooper, 1985): (a) una diagnosi descrittiva, che si basa sulla registrazione dei sintomi e dei segni, senza implicazioni eziologiche ed interpretazioni o ipotesi concernenti i meccanismi psicopatologici sottostanti (ad esempio: schizofrenia); (b) una diagnosi eziologica, in cui si fa riferimento alla (ipotizzata o dimostrata) causa dei disturbi (ad esempio: disturbo post-traumatico da stress); (c) una diagnosi interpretativa, nella quale vengono avanzate delle ipotesi circa $i$ meccanismi ed i processi psicologici considerati in atto (ad esempio: disturbo di personalità passiva-aggressiva). Tale differenziazione si riflette nella stessa nomenclatura diagnostica, in cui è possibile riscontrare livelli diversi di specificazione: così, accanto a categorie diagnostiche semplici definite solo in senso sindromico, e contrassegnate da una eziologia sconosciuta, dubbia o molteplice (ad esempio: sindromi affettive), vi sono categorie composte, definite anche sul piano causale grazie all'aggiunta di uno o più termini (ad esempio: disturbo post-traumatico da stress) (Scadding, 1988).

$\mathrm{Va}$ inoltre sottolineato che, al fine di minimizzare la varianza interpretativa tra differenti clinici relativamente all'importanza da attribuire alla presenza di certi sintomi, si è progressivamente imposta la necessità di una definizione operazionale attraverso la quale siano chiaramente specificate le costellazioni di sintomi necessarie e sufficienti per poter fare una determinata diagnosi.

Nella realtà, quindi, degli attuali sistemi classificatori psichiatrici, ivi compreso l'ICD-10, coesistono una classificazione ispirata a criteri di tipo sintoma- tologico e di tipo eziologico. Peraltro, l'opportunità di separare queste due dimensioni è stata tra le ragioni che hanno indotto a mettere a punto delle classificazioni multi-assiali, volte ad evitare che determinati convincimenti relativi all'eziologia di un disturbo potessero influenzare i giudizi a livello della fenomenologia sintomatologica (Kendell, 1984).

\section{Modelli di classificazione: empirica/inferenziale, monotetica/politetica}

In medicina si possono riconoscere due differenti tipi di approcci nella costruzione di un sistema classificatorio: un approccio empirico ed uno inferenziale. Il primo tipo di approccio è strettamente limitato ai fatti direttamente osservabili, e solo su quelli costruisce le proprie categorie classificatorie; il secondo, invece va al di là di quanto è strettamente osservabile ed avanza inferenze o ipotesi circa le possibili cause e gli ipotetici processi sottostanti ai fenomeni osservabili.

In questi ultimi anni, come ha sottolineato Feinstein (1972), nell'ambito dei sistemi classificatori in medicina (ed in psichiatria) è stato gradualmente preferito un approccio empirico rispetto a quello inferenziale, ed è stata inoltre attribuita una crescente importanza alla riproducibilità delle osservazioni $e$ delle diagnosi, grazie anche allo sviluppo di metodologie di analisi statistica ed all'uso di sistemi computerizzati. Peraltro, in campo psicopatologico si ha a che fare anche con processi intrapsichici, o tratti di personalità, che non sono di per sé osservabili; tali strutture o processi non osservabili direttamente non solo sono utili, ma possono essere necessari al fine di costruire una nosografia psicopatologica; a causa del loro carattere astratto ed ipotetico essi sono noti, in campo filosofico, come concetti aperti (Millon, 1991). Tuttavia, molti tra queste strutture o processi possono (e devono) essere definiti, ed in gran parte sono riducibili, ad un insieme di eventi di carattere empirico (quali, ad esempio, la descrizione fatta dal paziente, la presenza di specifici markers comportamentali, la risposta a test specifici, ecc.: si consideri il caso del meccanismo della proiezione, la cui esistenza può essere inferita sia dal fatto che la persona in questione attribuisce propri tratti ad altri, sia da una storia di comportamenti litigiosi, sia infine dalla risposta fornita a test specifici).

Qualsiasi sia la strategia impiegata, i sistemi classificatori ottenuti sono essenzialmente di due tipi:

- un sistema monotetico, in cui le classi differi- 
scono l'una dall'altra a seconda della presenza o meno di una singola variabile (o di un piccolo numero di variabili), che deve essere necessariamente presente per poter classificare la categoria in oggetto (questo è, ad esempio, il caso delle malattie infettive, che vengono differenziate l'una dall'altra quasi esclusivamente in relazione alla presenza o meno di un agente batterico o virale specifico);

- un sistema politetico, in cui i membri di una classe diagnostica hanno in comune molti elementi (ad esempio numerosi sintomi), ma non necessariamente tutti (e questo è il caso della maggior parte dei disturbi oggetto di indagine e di classificazione da parte delle varie specialità mediche, ivi compresa la psichiatria).

Nonostante che una classificazione di tipo monotetico si presti a minori ambiguità, di fatto i sistemi classificatori vigenti (ivi compreso l'ICD-10) sono in gran misura politetici, e permettono quindi una maggiore flessibilità di impiego nella pratica clinica.

\section{Classificazioni gerarchiche/multiassiali}

Nell'intero ambito della psicopatologia sono stati messi a punto vari modelli tassonomici volti ad ordinare questo campo di indagine secondo approcci predeterminati: tra essi, i due principali modelli (che non devono essere intesi come mutuamente esclusivi) sono rispettivamente di tipo verticale od orizzontale (Millon, 1991).

Il modello verticale, noto anche come modello gerarchico, organizza le varie categorie tassonomiche (ad esempio, schizofrenia, demenza o fobia) in una serie di livelli differenziati, nei quali i livelli più bassi sono sussunti come parti di quelli più elevati. Ad esempio, la diagnosi di un disturbo demenziale, a cui è attribuito un livello gerarchico molto elevato, preclude la diagnosi di fobia, a cui è riconosciuta una posizione gerarchica molto più bassa. Un'impostazione gerarchica nella classificazione del mondo naturale, originariamente proposta da Aristotele, è stata successivamente ripresa da Linneo in campo biologico, con la creazione di classi, ordini, famiglie, ecc., ordinate appunto gerarchicamente (Parshall \& Priest, 1993). In psicopatologia Jaspers (1964) è stato tra i principali sostenitori di un modello classificatorio di tipo gerarchico: il modello da lui proposto prevedeva un primo gruppo definito delle "malattie somatiche note con disturbi psichici"' (malattie cerebrali, malattie del corpo con psicosi sintomatiche, intossicazioni, ecc.); un secondo gruppo, denomina- to come "i tre circoli delle grandi psicosi" (epilessia genuina, schizofrenia, affezioni maniaco-depressive); ed un terzo gruppo, infine, chiamato gruppo delle psicopatie (nevrosi, personalità anormali, reazioni anormali). Va notato che l'ICD-9 aveva esplicitamente adottato un simile orientamento gerarchico; la tabella I, proposta da Cooper (1988), mostra come, utilizzando anche un criterio di tipo gerarchico, si possano condensare i 16 gruppi di disturbi compresi sia nell'ICD-9 che nell'ICD-10 (e nello stesso DSMIII-R) dapprima in 8 gruppi, quindi in 6 ed infine in 3. Ciascuna di tali condensazioni può essere impiegata al fine di illustrare, tra l'altro, le funzioni di vari operatori in differenti servizi assistenziali. Le classificazioni indicate come A e B sono indicate per gli operatori psichiatrici forniti di adeguato training ed esperienza; la classificazione $C$, che contiene solo 6 categorie, ha un livello intermedio di complessità. Infine, la classificazione D, semplificata al massimo, può essere adottata da operatori dell'assistenza sanitaria di base.

Uno sviluppo proprio dei sistemi gerarchici è rappresentato dalla loro progressiva organizzazione sotto forma di alberi decisionali, il che, per l'impiego nella pratica clinica, potrebbe costituire una rilevante conquista di siffatti sistemi: tuttavia, non è facilmente identificabile una struttura intrinseca della psicopatologia che permetta un ordinamento rigoroso e sequenziale dei vari disturbi in questo modo; inoltre, il fatto che gli attuali sistemi classificatori prevedano (ed anzi sollecitino) la possibilità di diagnosi multiple va contro l'adozione di un modello tassonomico verticale.

Un modello orizzontale è conosciuto come $\mathrm{mul}$ tiassiale: in esso varie classi di attributi (quali sintomi o fattori eziologici, o altri ancora) sono ordinati in una serie di categorie parallele o allineate. Come sottolinea Millon (1991), l'adozione di un siffatto modello orizzontale ha rappresentato un vero e proprio salto epistemologico nella pratica clinica, in quanto ha coinciso con il passaggio da un modello tassonomico centrato sulle malattie-infettive, in cui il compito del clinico era quello di chiarificare una situazione, identificando eventuali sintomi o circostanze di confondimento al fine di mettere in luce il meccanismo fisiopatologico di base, ad un modello in cui non esistono più sintomi o circostanze secondarie, o irrilevanti, ma nel quale invece si incoraggia la raccolta di tutte le informazioni possibili e la loro registrazione su assi specifici, che vengono ad acquisire un ruolo preciso sia dal punto di vista diagnostico che operativo (asse del disturbo psichiatrico, asse 
Tabella I. - Prospetto classificatorio dei principali disturbi psichiatrici secondo differenti livelli di specificazione.
1. Demenze
1. D. organici e sintomatici
2. D. transitori e sintomatici organici
1. D. organici e sintomatici
3. D. dovuti all'alcool
$\left\{\begin{array}{l}\text { 2. D. da abuso di alcool o di } \\ \text { sostanze }\end{array}\right.$
2. D. da abuso di alcool o di sostanze
4. D. dovuti all'abuso di sostanze
3. Schizofrenia e d. correlati
3. D. mentali gravi (psicotici)
5. D. schizofrenici
6. D. paranoidi, acuti ed altri
7. D. affettivi
4. D. affettivi
8. D. nevrotici
9. D. somatoformi
5. D. nevrotici e da stress
10. D. psicofisiologici
4. D. nevrotici, da stress e della personalità
1. D. mentali gravi (psicotici)
11. Reazicni da stress e d. dell'adat- tamento
12. D. della personalità
13. Altre anormalità del comporta- mento adulto, delle emozioni e degli impulsi
6. D. della personalità e del comportamento
14. D. con insorgenza nell'infanzia
o nell'adolescenta e durante lo
sviluppo
15. D. dello sviluppo
16. Ritardo mentale
7. D. dell'infanzia, adole- scenza e dello sviluppo
8. Ritardo mentale
5. D. dell'infanzia, adole- scenza e dello sviluppo
6. Ritárdo mentale
2. D. nevrotici, da stress e della personalità

Tratta da Cooper, 1988.

della personalità, asse di possibili concomitanti condizioni di malattia fisica, ecc.).

\section{NOTE EPISTEMOLOGICHE}

In medicina, così come in psichiatria, il modello epistemologico della conoscenza adottato, esplicitamente o talvolta implicitamente, dai clinici è stato in genere di tipo induttivista: secondo questo modello, nella conoscenza clinica si passava da proposizioni particolari (il caso clinico) a proposizioni universali (la diagnosi e la classificazione del caso). Pertanto al clinico veniva assegnato il compito di raccogliere, nella maniera più "obiettiva" possibile, il massimo numero di osservazioni, di classificarle ordinatamente e di formulare successivamente delle leggi di carattere generale, attraverso una delle molteplici attività logiche di tipo induttivo.

Tuttavia, negli ultimi anni, particolarmente sotto l'influenza del pensiero di Popper, è stata elaborata una visione del ragionamento scientifico, e quindi della stessa medicina e del ragionamento clinico, di tipo ipotetico-deduttivo, secondo cui le osservazioni "neutre", effettuate in assenza di una teoria o di un modello concettuale, non esistono e non possono esistere: come ha affermato Popper, «Le "osservazioni cliniche", come tutte le altre osservazioni sono interpretazioni alla luce di teorie, e unicamente per questo motivo esse sembrano sostenere le teorie alla cui luce vengono intese) (citato da Baldini, 1975). In ac- 
cordo con la visione ipotetico-deduttiva, la scienza, e l'osservazione clinica al pari, cominciano di fatto dalla formulazione di congetture, suggerite dallo scienziato posto di fronte ad un problema. Costui (al pari dell'uomo comune) possiede già nella propria mente una serie di teorie, o di modelli concettuali, frutto delle conoscenze precedenti o della elaborazione scientifica a lui preesistente; allorquando egli si trova dinanzi ad un problema nuovo, o inatteso, formula una ipotesi che cerca di sottoporre al controllo dei fatti: «Questa fase è eminentemente deduttiva poiché in essa lo scienziato deduce dalla propria ipotesi una serie di conseguenze osservabili e le "controlla osservando" se esse si siano o meno realizzate» (Federspil, 1980).

Non è questa la sede per una discussione approfondita dei problemi relativi all'epistemologia medica (di cui va con forza ribadita l'importanza, spesso misconosciuta), per cui si rimanda a specifiche opere sul tema (Baldini, 1975; Federspil, 1980; Scandellari, 1981; Scandellari \& Federspil, 1983); è sufficiente qui dire che, come hanno sottolineato Campbell (1987), Federspil (1980) e Wallace (1988), la verità sembra stare nel mezzo, in quanto nell'operare clinico-diagnostico quotidiano vengono utilizzati sia procedimenti di tipo induttivo che ipotetico-deduttivo, per cui l'osservazione dei fatti, la generazione di ipotesi, la loro confutazione o conferma si succedono e si intersecano inestricabilmente. Inoltre il peso relativo del procedimento induttivo o ipotetico-deduttivo sembra variare a seconda del momento considerato: così «Nella fase iniziale dell'iter clinico non è possibile negare la necessità di una raccolta molto estesa di fatti iniziali, effettuata in assenza di ipotesi diagnostiche consapevoli. Poiché i sintomi raccolti sono dotati di un valore segnaletico, essi permettono attraverso un ragionamento induttivo di prospettare ipotesi diagnostiche. In ciò quindi l'importanza dell'argomentazione induttiva non può non trovare un riconoscimento generale da parte dei medici [...] Nella fase avanzata dello studio clinico, molto spesso il medico giunge a formulare una serie di possibilità diagnostiche; in questo stadio il procedimento clinico prende il nome di "diagnostica differenziale" e si effettua controllando ed eliminando una ad una le varie ipotesi proponibili, o controllando e corroborando indefinitamente la teoria vera. Si deve riconoscere che questo processo avanza per "congetture e confutazioni" e che i dati sperimentali vengono ricercati come tentativi di falsificazione di teorie diagnostiche spesso in competizione. In questa evenienza quindi il ruolo e ed il valore della deduzione ap- paiono indiscutibili e insostituibili» (Federspil, 1980). In questo senso «il sapere clinico si costituisce all'intersezione di due aspetti diversi e complementari: un aspetto descrittivo e un aspetto interpretativo" (Lanteri-Laura \& Del Pistoia, 1983).

Nel procedimento diagnostico, la decifrazione dei sintomi e dei segni assume un ruolo cruciale (Barras, 1990): mentre i primi rappresentano una qualsiasi variazione evidenziabile nell'organismo ammalato, rilevabile o dal malato stesso o dal medico, i secondi sono riconosciuti ed interpretati solo grazie all'esistenza di un sapere medico preciso: l'esame clinico consiste appunto nella ricerca di segni (Lanteri-Laura, 1983), ed è guidato da un sapere e da un saperfare specifici. Ciascun segno è inizialmente polisemi$c o$, in quanto rinvia di solito a molteplici ipotesi diagnostiche (salvo che nel caso dei cosiddetti sintomi o segni patognomonici); tuttavia, nel momento in cui esso si congiunge ad altri segni, si riduce il suo alone semantico e esso acquista quindi un significato monosemico. Pertanto «Fare la diagnosi vuol dire sviluppare una teoria che trasforma i sintomi in segni, che decifra gli indizi e spiega i sintomi in quanto li trasforma in segni. Trasformare $i$ sintomi in segni vuol dire ricostruire la loro storia. Ma non si può ricostruire la storia dei sintomi se non si congettura sul loro significato" (Rosaia 1986). Inoltre ciascun segno ha, nella fase iniziale della valutazione diagnostica, soprattutto un valore informativo, rappresentato dalla sua idoneità a fornire indicazioni utili alla formulazione di una ipotesi diagnostica indipendentemente dalla sua positività o negatività, mentre in una fase successiva, allorquando il clinico cerca di confutare o corroborare l'ipotesi diagnostica, esso acquista un valore probativo grazie alla capacità del segno di comprovare l'esistenza di una determinata malattia (Scandellari, 1981). Foucault (1969) ha magistralmente analizzato l'importanza di questo processo per la fondazione della moderna medicina clinica, ed ha sottolineato l'importanza del passaggio da una "botanica dei sintomi ad una grammatica dei segni"'.

Nel caso più specifico della diagnosi, va ricordato quanto già affermò Murri circa mezzo secolo or sono, ossia che «In clinica non si tratta di conoscere, ma di riconoscere»: per questa ragione, la diagnosi è sempre una diagnosi differenziale, rappresentando una scelta operata tra diverse possibilità; in essa si procede, al contrario di quanto accade nelle scienze teoriche, dall'ignoto al noto. Inoltre, nella pratica clinica, si adottano spesso giudizi di tipo probabilistico: cosi «A ben guardare, nella grandissima mag- 
gioranza dei casi, il processo mentale del clinico che compie una diagnosi consiste nel valutare da un lato la probabilità che i segni riscontrati nel paziente indichino una certa malattia e dall'altro la probabilità che quella malattia sia effettivamente presente in quel malato" (Federspil, 1980); infatti, talora è impossibile giungere ad una verifica assoluta dell'ipotesi ma si devono purtuttavia adottare delle decisioni operative. Da questo punto di vista, il ben noto teorema di Bayes rappresenta una delle formalizzazioni della logica che permette di pervenire ad una valutazione razionale di un'ipotesi diagnostica data (Rosaia, 1986; Scandellari, 1981), e costituisce una delle procedure impiegate, consapevolmente o inconsapevolmente, da ciascun clinico nel proprio operare quotidiano.

\section{PROBLEMI ATTUALI DELLA CLASSIFICAZIONE E DELLA DIAGNOSTICA IN PSICHIATRIA}

\section{Modelli concettuali e diagnostici in psichiatria}

Negli ultimi decenni la massima parte degli operatori psichiatrici si sono ispirati a quattro modelli concettuali diversi: un modello biomedico, un modello psicologico, uno comportamentale ed uno sociale (Lazare, 1973), spesso in aperta antinomicità tra loro. Ciascuno di questi modelli implica un modello teorico differenziato di malattia mentale, un modello teorico-pratico di intervento ed un modello di interazione specifico tra coloro che sono coinvolti nella situazione considerata (medico/malato, psicoterapeuta/paziente, maestro/allievo, assistente sociale/assistito, ecc.) (Ponsi \& Bonner, 1979). Peraltro, all'interno di ciascuno di questi quattro modelli generali la presenza di ulteriori diffrazioni concettuali e dottrinarie (basti pensare all'esistenza di modelli psicogenetici fortemente differenziati) ha portato all'adozione di ulteriori sub-modelli interpretativi differenziati, e di sistemi classificatori altrettanto differenziati. La polarizzazione tra gli appartenenti a varie scuole di pensiero è stata facilitata dal privilegio spesso accordato ad un approccio inferenziale, piuttosto che empirico, nella costruzione di ciascun modello concettuale (e classificatorio), attraverso il quale si cercava soprattutto di confermare gli assunti di partenza piuttosto che sviluppare una seria e metodologicamente corretta indagine scientifica. Tutto ciò ha indotto alcuni autori ad asserire che la psichiatria si trova ancora in una situazione "preparadigmatica", secondo la nota terminologia kuhniana (Federspil, 1982; Pera, 1982).

Mentre non è questa la sede per una specifica ed estensiva discussione di tali tematiche, è comunque importante sottolineare che il concetto di malattia è eminentemente multidimensionale, non è riducibile ad una pura dimensione frequentistica (di qui la difficoltà di equiparare la normalità intesa in senso frequentistico con la normalità biologica e psichica), è definibile solo in rapporto alle capacità adattative dell'organismo, e la malattia stessa può essere indagata da molteplici punti di vista e secondo molteplici prospettive, che non sono mutuamente esclusive (Berlinguer, 1982; Blois, 1988; Federspil, 1990). Pertanto, «Tutte le argomentazioni basate su un supposto "modello medico di malattia" sono sospette, dal momento che la nosologia attuale include malattie definite secondo criteri di differente natura, che hanno differenti implicazioni fattuali» (Scadding, 1988).

In campo psichiatrico la difficoltà di giungere ad un paradigma unificante svela anche la difficoltà di definire, in maniera scientificamente corretta ed operativamente utile, la nozione di salute mentale, che non può essere dedotta da quella di malattia mentale nè essere semplicisticamente interpretata come mera assenza di malattia (de Girolamo, in stampa).

Inoltre, è difficile pensare, al momento, ad una tassonomia psichiatrica che sia del tutto avalutativa, ossia che prescinda del tutto da una assunzione di valori e che anche su questi orienti la propria classificazione (Scadding, 1988). L' importanza di questa asserzione per la fondazione stessa della nosografia psicopatologica è stata paradossalmente dimostrata da Bentall (1992), il quale ha messo in evidenza come, in una prospettiva rigorosamente avalutativa, la stessa felicità possa essere classificata come un disturbo psichiatrico (sotto la denominazione di disturbo affettivo maggiore, tipo piacevole), in quanto essa soddisfa molti dei criteri impiegati per identificare la maggior parte delle malattie mentali (è statisticamente anormale; consiste in un cluster discreto di sintomi; vi è qualche evidenza che rifletta un anormale funzionamento del sistema nervoso centrale, ed è associata con alcune anormalità cognitive, in particolare una mancanza di contatto con la realtà).

$\mathrm{Va}$ anche detto che la nozione stessa di classificazione e la necessità di disporre di un apparato diagnostico in campo psichiatrico sono state messe in discussione da molti autori: è stata così enfatizzata la dimensione della clinica come "scienza dell'indivi- 
duale" (Federspil, 1980), nel cui ambito non esisterebbero leggi a carattere generale, ogni fenomeno rappresenterebbe un caso a sè, le generalizzazioni risulterebbero impossibili e da rifiutare, le persone sarebbero incommensurabili tra loro ed in perenne divenire, ecc. In campo psichiatrico tali posizioni critiche sono state accentuate da coloro che denunciavano nella diagnosi sia un rischio di reificazione del paziente e della sua sofferenza che uno strumento di possibile stigmatizzazione, mettendo in evidenza il carattere artifattuale delle diagnosi psichiatriche (Szasz, 1991).

Mentre tali obiezioni hanno avuto comunque il merito di sottolineare sia la necessità di disporre di definizioni chiare ed esplicite che di investigare in maniera accurata e riflessiva i singoli casi, evitando le facili omologazioni (a cui in passato si accompagnava una applicazione seriale di trattamenti standard a grandi numeri di pazienti istituzionalizzati), se tale approccio fosse adottato in maniera rigida, esso determinerebbe la pratica impossibilità di comunicare tra operatori (ed utenti) differenti e di apprendere dall'esperienza.

\section{Il dilemma categoriale/dimensionale}

In questi ultimi anni si è intensificato il dibattito tra i fautori, nel campo della psicopatologia, di un approccio di tipo categoriale, attraverso il quale le categorie diagnostiche sono ben differenziate qualitativamente, separate tra loro e mutuamente esclusive, ed un approccio di tipo dimensionale, attraverso cui le specifiche entità morbose vengono considerate come deviazioni quantitative di un continuum relativo alla personalità, alla percezione, alla cognizione, all'umore, ecc. In generale, come ha sottolineato Hempel (1968), in campo scientifico si è assistito ad una progressiva evoluzione da modelli di tipo dicotomico (ossia categoriali), in cui alcune specifiche variabili sono considerate presenti o assenti, a modelli di tipo continuo (ossia dimensionali), in cui vi è un continuo di gradazioni diverse della stessa dimensione indagata, il che permette una migliore precisazione delle caratteristiche distintive della condizione oggetto di indagine.

Kendell (1989) e Goldberg \& Huxley (1993) hanno con chiarezza descritto le strategie che dovrebbero essere impiegate al fine di validare le categorie incluse in un sistema classificatorio categoriale:

(a) identificazione e descrizione della sindrome, sia attraverso l'osservazione clinica che attraverso tecniche specifiche di analisi dei cluster sintomatologici; nel confronto tra sistemi classificatori ed osservatori diversi, le varie categorie dovrebbero inoltre mostrare una sufficiente attendibilità;

(b) identificazione dei confini, o dei punti di rarefazione, tra sindromi diverse, ottenuta attraverso tecniche di analisi statistica specifiche (analisi discriminante, analisi dei tratti latenti); identificazione, inoltre, di reali discontinuità tra esse e la "normalità"';

(c) effettuazione di studi di follow-up volti sia a stabilire un decorso ed un esito specifici per ciascun disturbo o sindrome, che ad evidenziare una soddisfacente stabilità nel tempo di ciascuna diagnosi;

(d) effettuazione di trial terapeutici volti a mettere in luce precise risposte al trattamento, differenziate per le varie categorie diagnostiche;

(e) effettuazione di studi di tipo familiare, al fine di evidenziare una familiarità del disturbo o della sindrome considerata;

(f) infine dimostrazione dell'esistenza di una specifica associazione tra il disturbo o la sindrome in oggetto ed un qualche tipo di anormalità (di tipo anatomo-istologico, psicologico, biochimico o molecolare).

È evidente che queste condizioni sono, a tutt'oggi, difficilmente soddisfatte, soprattutto nel caso dei "disturbi emotivi comuni" (disturbi depressivi, disturbi d'ansia, disturbi di personalità, ecc.), per cui, a parere di numerosi autori, un approccio dimensionale si presta meglio alla loro classificazione, mentre un approccio categoriale si adatta in maniera soddisfacente alla classificazione dei disturbi più severi (quali le psicosi), che meglio soddisfano i criteri sopra enunciati. Tuttavia è stata gradualmente riconosciuta l'importanza euristica di modelli di tipo continuo, o dimensionale, anche nel campo della fenomenologia psicotica, poiché pure sintomi considerati al di fuori del range dei fenomeni "normali", quali ad esempio le allucinazioni, possono in realtà presentarsi con una molteplicità di sfumature e di gradazioni, piuttosto che come fenomeni tutto-o-niente, per cui, ai fini di una loro accurata descrizione, i modelli dimensionali potrebbero apparire più adeguati (de Girolamo, 1991).

Comunque, in termini di impiego nella pratica clinica, ciascuna delle due strategie classificatorie ha i propri vantaggi ed i propri svantaggi: per quanto riguarda l'approccio categoriale, i primi sono rappresentati dalla semplicità di impiego nella clinica e nel training degli operatori, dalla facilità di utilizzo in campo informatico, dalla idoneità per la ricerca 
epidemiologica e dalla possibilità di utilizzare delle gerarchie diagnostiche. Peraltro un approccio di tipo categoriale comporta anche numerosi svantaggi, quali la difficoltà di classificare correttamente i casi che sono al "confine" tra differenti categorie, la perdita di pazienti che sono al di sotto della soglia stabilita per poter far diagnosi, la necessità di dover utilizzare categorie ibride, quali atipico o misto, ed infine l'uso di scale nominali piuttosto che ordinali. Un approccio di tipo dimensionale, invece, riduce l'uso di etichette diagnostiche, con il rischio di stigmatizzazione ad esse correlato, e facilita la classificazione di casi situati al confine tra differenti categorie, dei casi difficili e della comorbidità. Peraltro esso è certamente più problematico per l'impiego nella pratica clinica quotidiana e rende le comparazioni più ardue.

Nelle vigenti classificazioni, comunque, come si procede verso un livello di maggiore specificazione diagnostica, si passa gradualmente (ed inevitabilmente) da un approccio di tipo categoriale ad uno dimensionale; ad esempio, nell'ICD-10 la diagnosi di episodio depressivo corrisponde effettivamente ad una categoria diagnostica precisabile e definita qualitativamente; tuttavia, nel momento in cui si cerca di operare una ulteriore precisazione diagnostica, nel senso della creazione di sottocategorie (ad esempio: episodio depressivo lieve o di media gravità), si adotta un criterio quantitativo implicito di gravità, quindi dimensionale (Millon, 1991). In questo senso, le sottocategorie vanno interpretate come un tentativo di incorporare caratteristiche quali gravità, decorso e presenza di caratteri aggiuntivi in un sistema diagnostico categoriale (Goldberg \& Huxley, 1993). Di recente Fava e Kellner (1993) hanno proposto di incorporare apertamente, nei sistemi classificatori psichiatrici, un criterio di "stadiazione" dei diversi disturbi, al pari di quanto si fa comunemente in molti altri settori della medicina e di quanto, di fatto, avviene anche nei sistemi classificatori psichiatrici vigenti attraverso la creazione di sotto-categorie; l'utilizzazione di una stadiazione consentirebbe di precisare meglio la gravità, l'estensione ed altre caratteristiche del disturbo in esame.

\section{Finalità e storicità della diagnosi}

Come si visto sopra, la classificazione, e la sua traduzione operativa rappresentata dalla diagnosi, hanno un insostituibile valore cognitivo; tuttavia, soprattutto nella pratica psichiatrica e nell'articolazio- ne del rapporto medico-paziente, una delle funzioni della diagnosi è anche quella di fornire un nome, 0 una etichetta, ad un insieme di comportamenti vissuti come diversi, inesplicabili ed inquietanti, dando così un senso ad esperienze problematiche ed assimilandole ad un ordine familiare:

«il clinico desidera la certezza e la sensazione di controllo della situazione che deriva dal dare un nome al problema. In un certo senso, anche il paziente vuole la sensazione di controllo che gli deriva dal fornire un nome al problema. Una diagnosi serve pertanto a rimuovere il mistero del problema attribuendogli un nome dal quale derivano una serie di considerazioni inerenti al trattamento, alla cura, alle implicazioni sociali e personali del problema stesso, ed all'accettazione sociale delle proprie diminuite capacità. Tuttavia, per i pazienti la diagnosi è meno importante di una ampia comprensione dei loro problemi e di quanto può essere fatto per loro» (Brown, 1990).

In questo senso, come ha sottolineato lo stesso autore (Brown, 1990), una critica della diagnosi coincide con una critica della psichiatria stessa, in quanto la diagnosi è il linguaggio della psichiatria e ne definisce i limiti operazionali. Inoltre, proprio intorno alla costruzione, alla critica ed al superamento di intere categorie diagnostiche e di speficici modelli nosografici si articola l'intera vicenda storica della psichiatria come costruzione sociale e non solo come ambito di esplorazione scientifica "neutra": le vicende dell'isteria (Llyod, 1986), del disturbo posttraumatico da stress (de Girolamo, 1993), della sindrome premestruale (de Girolamo et al., 1989), del disturbo da attacchi di panico (Hollister, 1992; Terzian, 1993), ed addirittura dell'intera nozione di "nevrosi"', oggi accantonata, rappresentano esempi paradigmatici di questo assunto, e devono essere oggetto di accorta riflessione nell'ambito di qualsiasi discussione relativa alla nosografia psichiatrica.

\section{Variabili che influenzano e condizionano il processo diagnostico e la sua attendibilità}

Soprattutto nella pratica clinica quotidiana, laddove non sono utilizzati strumenti standardizzati di valutazione, il clinico basa le sue decisioni diagnostiche (e quindi, in buona misura, anche le sue scelte terapeutiche) non sull'informazione di per sè, ma sulla sua percezione di tali informazioni (Williams, 1979), percezione che, in quanto tale, può essere influenzata da molteplici fattori.

È stato infatti ripetutamente dimostrato che variabili quali il sesso, l'età, la razza, il livello socio-e- 
conomico e culturale, lo stesso aspetto fisico del paziente, il setting in cui viene effettuata la valutazione, oltre a rappresentare informazioni che modificano le valutazioni probabilistiche del clinico (ad esempio, numerosi disturbi sono più frequenti in un sesso rispetto all'altro, $o$ in persone di una specifica fascia d'età o di una specifica razza), possono anche influenzare e distorcere in maniera significativa le conclusioni diagnostiche del clinico, condizionando lo stesso livello di accordo diagnostico tra clinici diversi. Ad esempio, in una recente ricerca effettuata allo scopo di valutare il peso di due tra queste variabili (sesso e razza) nel ragionamento diagnostico, si è visto che il livello di accordo diagnostico tra clinici diversi era molto maggiore allorquando non veniva fornita alcuna informazione sul sesso e la razza dei pazienti considerati (Loring \& Powell, 1988). Quando tale informazione era fornita, i clinici tendevano a fare una diagnosi corretta soprattutto quando il sesso e la razza del paziente descritto erano simili alla propria.

Il discorso relativo alla diagnosi in psichiatria rimanda quindi anche a quello relativo alle modalità decisionali che prevalgono nel lavoro clinico: in tal senso, le (poche) ricerche condotte sembrano dimostrare soprattutto due cose: «la fiduciosa rapidità con cui si prendono decisioni diagnostiche e l'insufficiente nozione che lo psichiatra ha dei temi o momenti cruciali nella raccolta delle informazioni» (Cooper, 1984). Ad esempio, in due studi relativi a dei colloqui filmati, è emerso che gli psichiatri traevano impressioni diagnostiche abbastanza definite entro il primo minuto del colloquio (Gauron \& Dickinson, 1966, 1969). Nel secondo di questi due studi, è anche emerso che l'impressione ricavata dalla visione del colloquio registrato con il paziente contava molto di più delle notizie anamnestiche al fine di raggiungere una decisione diagnostica: alcuni markers comportamentali, ritenuti dai clinici partecipanti alla ricerca come altamente indicativi e predittivi di una determinata diagnosi, apparivano infatti decisivi nell'orientare, con estrema rapidità, l'orientamento dei clinici stessi. L'importanza di queste considerazioni risiede nel fatto che gli stessi tratti comportamentali possono essere in accordo con varie ipotesi diagnostiche (hanno cioè un elevato valore polisemico), e tale eterogeneità era appunto alla base della variabilità diagnostica riscontrata negli studi citati. In una ricerca simile, realizzata ancora attraverso l'uso di colloqui clinici filmati, Sandifern et al. (1970) hanno dimostrato che, dopo solo tre minuti di proiezione, gli psichiatri esaminati erano già arri- vati ad una diagnosi iniziale e questa era, in tre quarti dei casi, identica alla diagnosi finale, formulata in media dopo circa 30 minuti di proiezione.

Inoltre le attese e le aspettative dell'osservatore possono altrettanto influenzare e predeterminare le conclusioni diagnostiche; lo studio condotto da Temerlin (1968) è paradigmatico al riguardo. In questo esperimento fu presentata a tre gruppi di psichiatri e psicologi clinici una intervista diagnostica registrata con un attore accuratamente istruito a recitare un racconto di assoluta "normalità". Tuttavia, prima dell'ascolto dell'intervista stessa, al primo ed al secondo gruppo fu lasciato ascoltare un commento casuale di un autorevole esperto: costui, nel primo caso affermò che il caso riguardava una "persona apparentemente nevrotica ma in realtà psicotica", mentre nel secondo caso asserì trattarsi di "un caso molto raro, in realtà di una persona perfettamente sana". Al terzo gruppo non venne invece fatto sentire alcun commento. Dopo l'ascolto della registrazione, fu chiesto a ciascuno di formulare una ipotesi diagnostica tratta da un elenco comprendente 10 psicosi, 10 nevrosi e 10 tipi di personalità, inclusa la personalità "normale o sana". Tutti i componenti del secondo gruppo diagnosticarono una personalità normale o sana; 12 (su 21) del terzo gruppo diagnosticarono una condizione di normalità, mentre nel primo gruppo (comprendente 25 psichiatri, 25 psicologi e 45 studenti di psicologia clinica) solo 8 intervistati affermarono che il soggetto era mentalmente sano; 60 formularono una diagnosi di nevrosi e $27 \mathrm{di}$ psicosi. In maniera imprevista emerse anche che $i$ più suggestionabili erano proprio gli psichiatri: infatti il $60 \%$ di essi formulò una diagnosi di psicosi, di contro al $28 \%$ degli psicologi clinici; in assoluto $i$ meno suggestionabili apparvero essere gli studenti di psicologia clinica. Inoltre nessuno tra gli psichiatri considerò la persona in esame come psicologicamente normale. Allorquando fu chiesto a ciascuno di spiegare in termini strettamente comportamentali e descrittivi le ragioni della propria scelta diagnostica, la maggior parte di coloro che avevano fatto diagnosi di nevrosi o psicosi giustificò la propria decisione sulla base di inferenze piuttosto che nei termini richiesti.

Sembrano quindi intervenire, nel ragionamento clinico, degli specifici meccanismi cognitivi che tendono a far selezionare le informazioni in maniera tale da renderle compatibili con una determinata ipotesi diagnostica o con una determinata gestalt conoscitiva, grazie ai quali la diagnosi può talora assumere il significato di "profezia autoavverantesi"; inoltre il 
privilegio accordato, nella valutazione diagnostica, alle inferenze facilita le distorsioni nella valutazione.

Si deve pertanto concludere che esiste, nella pratica diagnostica quotidiana, una elevata soggettività ed arbitrarietà dei giudizi - che si riscontra peraltro anche nelle specialità mediche considerate più "obiettive", quali la radiologia, la cardiologia, ecc. (Eisenberg, 1983) - e che numerose variabili extracliniche influenzano significativamente il processo diagnostico e la sua attendibilità; l'importanza ed il peso di queste variabili mutano con il mutare del setting in cui viene operata la valutazione e con il mutare del disturbo in questione, essendo molto meno rilevanti allorquando la costellazione di sintomi presentata dal paziente è chiara e l'eziologia ben determinata (come ad esempio nei disturbi a manifesta eziologia organica); la generale variabilità diagnostica tende invece ad accrescersi nelle situazioni più controverse $o$ più difficili sul piano diagnostico, ponendo talora problemi di non agevole soluzione.

\section{ATTENDIBILITÀ E VALIDITÀ DELLE DIAGNOSI PSICHIATRICHE}

«Gli esseri umani sono spesso illogici ed inefficienti nell'uso delle informazioni, fanno di frequente delle deduzioni erronee, ignorano l'evidenza, iperenfatizzano delle caratteristiche irrilevanti, e difettano di consistenza nelle valutazioni, sia con gli altri che con se stessi») Editoriale di Lancet (1987)

Come si è ora visto, un cospicuo numero di variabili influiscono sul processo diagnostico in psichiatria, aumentando la variabilità del giudizio clinico e compromettendo quindi l'attendibilità (reliability) delle diagnosi poste: in questo senso i processi cognitivi operanti nel ragionamento diagnostico sono spesso fallaci, come l'Editoriale di Lancet (1987) sopra citato, dedicato alla logica della diagnosi, afferma con chiarezza.

Nel campo della diagnosi psichiatrica le principali fonti di inattendibilità diagnostica, che sono all'origine del disaccordo diagnostico che si può riscontrare tra clinici diversi, possono essere suddivise in cinque categorie principali, tecnicamente definite come fonti di variabilità (Spitzer et al., 1978):

(a) una variabilità legata al soggetto, che si manifesta allorquando un paziente mostra un quadro clinico differente in momenti diversi: ad esempio un paziente ricoverato per una intossicazione alcolica acuta può, dopo alcuni giorni, sviluppare un "delirium tremens";

(b) una variabilità legata all'occasione della valutazione diagnostica, che emerge allorquando un paziente esibisce fasi diverse della stessa condizione morbosa in momenti diversi: si pensi ad un paziente con un disturbo bipolare, il quale può essere depresso in un determinato momento e maniaco in un tempo successivo;

(c) una variabilità legata all'informazione, che deriva dal fatto che clinici diversi possono utilizzare fonti di informazione diverse per valutare lo stesso paziente: ad esempio, un clinico puo fare domande ad un paziente relative ad aspetti specifici della sua condizione, che invece un altro clinico può non porre non ritenendole pertinenti, o rilevanti; o ancora un clinico può ottenere informazioni dai familiari del paziente, mentre un altro no;

(d) una variabilità legata all'osservazione, che si produce allorquando clinici diversi, confrontati con la stessa situazione, differiscono tra loro rispetto a quel che notano; ad esempio, può esservi un disaccordo relativamente al riscontro o meno di esperienze allucinatorie, o rispetto ad un tratto della personalità del paziente;

(e) infine una variabilità legata ai criteri diagnostici utilizzati, in particolare relativa ai criteri diagnostici di esclusione o inclusione (espliciti o impliciti) utilizzati per arrivare a formulare una ipotesi diagnostica.

Come hanno sottolineato Spitzer et al. (1978), le prime due fonti di disaccordo diagnostico, rappresentate dalla variabilità legata al soggetto ed all'occasione della valutazione diagnostica, riflettono dei fenomeni reali, oggettivabili, e non dovrebbero essere ignorate. La variabilità legata all'informazione può invece essere superata (o perlomeno ridotta grandemente) migliorando le capacità di valutazione diagnostica e di osservazione dei clinici, e soprattutto facendo ricorso a tecniche di valutazione clinica standardizzata, quali le interviste strutturate. A questo proposito va sottolineato che, insieme all'ICD-10, proprio al fine di favorire una applicazione uniforme dei criteri diagnostici in esso contenuti, sono state messe a punto ben tre interviste strutturate, rappresentate dalla Composite International Diagnostic Interview (CIDI) (Robins et al., 1988), dalla Schedules for Clinical Assessment in Neuropsychiatry (SCAN) (Wing et al., 1990) (che incorpora la decima edizione del "Present State Examination") ed infine dalla International Personality Disorder Exa- 
mination (IPDE) (Loranger et al., 1991). Queste interviste, che hanno scopi e strutture diverse (per una più precisa descrizione di questi strumenti si rinvia ad una recente review di Pull e Wittchen [1991]) ed utilizzano specifici algoritmi diagnostici, consentono di porre diagnosi secondo le categorie sia dell'ICD-10 che del DSM-III-R. La importanza, ai fini di una soddisfacente attendibilità diagnostica, di questi strumenti di valutazione standardizzata appare certa; non vi è dubbio che la loro messa a punto rappresenti uno dei fattori-chiave che sono all'origine dei maggiori progressi conseguiti dalla ricerca epidemiologica negli ultimi 20 anni (Robins, 1990).

Infine, la variabilità legata ai criteri diagnostici può essere diminuita solo attraverso l'uso di criteri diagnostici di inclusione ed esclusione precisi - come quelli contenuti negli attuali sistemi diagnostici (ICD-10 o DSM-III). La attendibilità diagnostica tende comunque a variare in funzione delle categorie diagnostiche considerate, essendo in genere più elevata nei disturbi ad eziologia organica rispetto ai disturbi psicotici funzionali, ed in questi ultimi rispetto ai disturbi nevrotici o della personalità (Kendell, 1974). Inoltre, allorquando si impiegano misure percentuali di concordanza, il loro significato muta notevolmente in rapporto al numero totale di categorie utilizzate; così una concordanza del $60 \%$ riferita a 40 categorie diagnostiche ha un significato ben diverso che se riferita a sole 4 categorie. Il livello di concordanza che emerge tra clinici diversi nella valutazione dei sintomi psichiatrici sembra essere nettamente superiore a quello relativo alla formulazione della diagnosi complessiva (Shepherd et al., 1968). Va tuttavia sottolineato (Meehl, 1985) che gli indicatori più attendibili non necessariamente rappresentano quelli diagnosticamente più importanti: ad esempio, la misurazione della pressione sanguigna costituisce un indicatore diagnostico ben più importante della misurazione antropometrica del torace, nonostante che l'attendibilità della prima sia molto più bassa se paragonata all'attendibilità del secondo tipo di misurazione.

Dopo aver discusso i problemi legati all'attendibilità delle diagnosi psichiatriche, ed alle procedure volte ad accrescerne la riproducibilità, restano tuttavia aperti i problemi legati alla validità delle categorie diagnostiche impiegate, che sono in parte indipendenti dai problemi di attendibilità e di accordo diagnostico: infatti, come ha scritto Feinstein (1985), due osservatori che, nel descrivere la pioggia, si riferiscano entrambi ad essa giudicandola neve, pur essendo tra loro in accordo (manifestando quindi una elevata inter-attendibilità), non possono cionondimeno essere considerati come dei credibili metereologi! Pertanto una misura che non ha una sua intrinseca validità è priva di utilità pratica (Grove et al., 1981).

Sono stati identificati diversi tipi di validità: (a) validità di costrutto, rappresentata dalla dimostrazione che determinati aspetti del quadro psicopatologico misurabili obiettivamente (come l'insonnia che accompagna la depressione, o l'ansia) si riscontrano effettivamente in presenza di diagnosi che li prevedano e non in presenza di diagnosi che li escludano; (b) validità di contenuto, costituita dalla dimostrazione che le caratteristiche che definiscono un dato disturbo o una sindrome vengono effettivamente identificate e poste in evidenza indipendentemente, prima che la diagnosi stessa venga posta; (c) validità concomitante, rappresentata dalla possibilità di giungere alla stessa diagnosi utilizzando due tecniche diagnostiche indipendenti (ad esempio una valutazione clinica ed un test); ed infine (d) validità predittiva, secondo cui la diagnosi consente di fare delle predizio$\mathrm{ni}$, in termini di prognosi, risposta al trattamento, decorso, ecc., che vengono successivamente confermate; delle quattro è tuttavia quest'ultima dimensione che riveste la massima importanza: infatti «nel contesto della psichiatria clinica, giudizi circa la validità diagnostica sono essenzialmente giudizi circa il valore predittivo, e di conseguenza circa l'utilità pratica» (Kendell, 1989), e «la diagnosi, come qualsiasi altra ipotesi, si prova sulle sue conseguenze» (Antiseri, 1983).

Sopra sono già state discusse le strategie utilizzabili al fine di validare l'esistenza di categorie di disturbi psichiatrici diversi, ed è stato messo in luce come lo stato attuale delle conoscenze in quest'area sia in gran parte insoddisfacente. In particolare, la mancanza di criteri di validazione esterni (ossia indipendenti dall'osservatore) per le varie categorie diagnostiche (rappresentati, ad esempio, da test di laboratorio o da indagini strumentali specifiche) ha rappresentato (e tuttora rappresenta) uno dei principali ostacoli frapposti alla validazione delle differenti categorie diagnostiche.

Inoltre, sino a non molti anni or sono, è stato ben visibile in psichiatria (e per molti disturbi ancora lo è) uno iato tra numerosità e specificità dei sistemi classificatori e diagnostici e sostanziale povertà ed uniformità delle strategie di trattamento. In altre parole, a diagnosi differenziate e spesso minuziose corrispondevano trattamenti pressocché identici, a dimostrazione di un ridotto potere predittivo delle diagnosi psichiatriche relativamente alle strategie tera- 
peutiche e di una relazione diagnosi-trattamento debole e non univoca (Williams, 1979). In particolare, la risposta ai diversi trattamenti farmacologici disponibili sino ad oggi non tende ad escludersi reciprocamente, come invece accade per le categorie diagnostiche stesse (Kendell, 1974); inoltre la presenza di sintomi specifici, o di determinate costellazioni di sintomi, è apparsa, in numerose ricerche, ben più importante della diagnosi in quanto tale nell'orientare la scelta del trattamento (Williams, 1979). Bisogna quindi sottolineare che i progressi che si conseguiranno attraverso la messa a punto di trattamenti specifici per ciascuno dei principali disturbi psichiatrici (Sartorius et al., 1993a) forniranno anche evidenze decisive per una validazione della intera nosografia psichiatrica.

\section{L'IMPORTANZA DELLA DIAGNOSI PER LA RICERCA EPIDEMIOLOGICA}

Nella prospettiva epidemiologica la diagnosi appare essenziale ed imprescindibile: infatti mutamenti nelle attitudini e nelle pratiche diagnostiche determinano, o possono determinare, mutamenti altrettanto rilevanti in termini di riscontri epidemiologici. A dimostrazione di questo assunto basta richiamare, tra le tante, tre ricerche di particolare rilievo.

La prima è rappresentata dal progetto diagnostico statunitense-inglese (Cooper et al., 1972). In questo studio, realizzato alla fine degli anni ' 60 , due team di ricercatori analizzarono 250 pazienti ricoverati consecutivamente in due ospedali psichiatrici rispettivamente a Londra ed a New York. Ciascun paziente fu valutato indipendentemente da uno degli psichiatri partecipanti al progetto, che utilizzava una procedura di valutazione standardizzata, e da uno psichiatra della locale istituzione, che esaminava invece il paziente secondo la propria prassi clinica di routine. Al termine di queste due valutazioni emerse che, mentre non vi erano significative differenze tra le percentuali di pazienti diagnosticati dai ricercatori come sofferenti di un disturbo schizofrenico o affettivo nei due ospedali, si rilevavano invece significative differenze con le percentuali di diagnosi effettuate dagli psichiatri locali. In particolare a New York la diagnosi di schizofrenia era due volte più frequente rispetto alla diagnosi standardizzata fatta dai ricercatori del progetto, mentre la diagnosi di depressione era quattro volte meno frequente ed addirittura la diagnosi di mania era dieci volte meno comune.
Al fine di allargare ulteriormente l'area dell'indagine, vennero anche comparate le percentuali delle più importanti categorie diagnostiche riscontrabili in 9 ospedali psichiatrici di New York con quelle prevalenti in 9 ospedali psichiatrici dell'area metropolitana di Londra. Emerse ancora che gli psichiatri statunitensi diagnosticavano molto più spesso la schizofrenia e molto più raramente la depressione psicotica e la mania. Il rapporto tra la diagnosi di schizofrenia e quella di disturbo maniaco-depressivo era di 12 ad 1 negli ospedali di New York, di contro ad un rapporto di 1 ad 1 negli ospedali londinesi. Tuttavia, allorquando venivano utilizzati i criteri diagnostici standardizzati messi a punto per la ricerca, tali differenze si riducevano grandemente sino a scomparire quasi del tutto. In sostanza, gli psichiatri americani diagnosticavano come schizofrenici pazienti che sarebbero stati considerati in Europa come affetti da un disturbo di tipo maniaco-depressivo, e ciò a causa di un approccio iperinclusivo nella diagnosi dei disturbi schizofrenici che prevaleva a quel tempo negli Stati Uniti. È interessante notare che, in una ricerca condotta circa 20 anni dopo in uno degli ospedali statunitensi partecipanti all'indagine originaria, sono stati messi in evidenza analoghi, elevati livelli di variabilità diagnostica da parte degli psichiatri operanti nell'istituzione (Lipton \& Simon, 1985). In particolare, in soli 16 degli 89 casi $(18 \%)$ in cui era stata fatta diagnosi di schizofrenia da parte degli psichiatri curanti, tale giudizio venne confermato dai ricercatori che applicavano rigorosamente i criteri diagnostici del DSM-III.

Il secondo studio da considerare è lo studio della WHO International Pilot Study of Schizophrenia (IPSS) (WHO, 1979). In questo progetto multicentrico, condotto in 9 paesi, furono inclusi pazienti schizofrenici diagnosticati secondo criteri standardizzati basati sull'ICD-8. Comparando le diagnosi fatte dagli psichiatri in ciascun centro collaborativo rispetto a quelle ottenute secondo il metodo standardizzato proprio della ricerca, emerse che la diagnosi di psicosi, e particolarmente quella di schizofrenia, era effettuata in maniera simile sia nei paesi in via di sviluppo che nei paesi sviluppati. Evidenti discrepanze si rilevarono, invece, rispetto all'approccio diagnostico adottato dagli psichiatri americani e russi. Infatti una rilevante percentuale dei pazienti diagnosticati come schizofrenici negli Stati Uniti ed in Unione Sovietica venivano considerati come affetti da un disturbo maniaco-depressivo o nevrotico negli altri centri. In sostanza i dati ottenuti nell'IPSS confermavano ed ampliavano quelli ottenuti nel progetto 
diagnostico statunitense-inglese sopra descritto.

Per concludere, in una ricerca condotta in uno dei più grandi ospedali universitari statunitensi (Loranger, 1990), confrontando le diagnosi fatte a 5143 pazienti negli ultimi cinque anni precedenti all'introduzione del DSM-II con quelle assegnate a 5771 pazienti nei primi cinque anni successivi alla pubblicazione del famoso testo americano, è stato rilevato un marcato decremento nella diagnosi di schizofrenia (dal $25 \%$ al $13 \%$ ), insieme con un massiccio aumento nel numero dei casi di disturbi della personalità e di disturbi affettivi. A parere dell'autore, è verosimile che molti dei casi diagnosticati secondo il DSM-III come schizofreniformi $(1 \%)$, psicosi atipiche $(1,5 \%)$, psicosi reattive $(0,5 \%)$ e disturbo di personalità schizotipico $(2 \%)$ avrebbero ricevuto una diagnosi di schizofrenia utilizzando i criteri diagnostici propri del DSM-II. Tuttavia tutte queste nuove categorie rappresentano, globalmente, solo il $5 \%$ del totale del campione, e spiegano pertanto solo in piccola parte il declino della diagnosi di schizofrenia. Il grande incremento nella diagnosi dei disturbi affettivi appare essere l'altra ragione della diminuzione suddetta: infatti il tasso della diagnosi di depressione unipolare aumentò dal $15 \%$ al $25 \%$, con un incremento del $67 \%$, mentre quella di disturbo bipolare dal $7 \%$ al $11 \%$, con un incremento del $55 \%$. Cio sembra confermare la già notata tendenza, da parte degli psichiatri americani, in epoca pre-DSM-III, a diagnosticare come casi di schizofrenia quei quadri sintomatologici per i quali gli psichiatri europei avrebbero invece fatto diagnosi di disturbo affettivo.

Questi tre studi, quindi, dimostrano con chiarezza che la possibilità di disporre sia di un sistema classificatorio omogeneo, coerente e consensualmente legittimato, che di procedure diagnostiche comparabili ed attendibili rappresenta una conditio sine qua non per l'intera ricerca epidemiologica.

\section{LA CLASSIFICAZIONE INTERNAZIONALE DELLE MALATTIE: L'ICD-10}

La necessità di disporre di un adeguato sistema classificatorio in campo medico, al fine di poter uniformare la nomenclatura, i criteri diagnostici e prognostici e rendere possibile sia l'effettuazione di ricerche internazionali che la comparabilità di quelle condotte su scala nazionale, portarono già nel 1893 alla adozione, da parte dell'Istituto Internazionale di
Statistica, della $1^{\text {a }}$ Classificazione Internazionale delle Cause di Morte. Fu previsto sin da allora di aggiornare tale classificazione ogni $10 \mathrm{anni}$, al fine di renderne possibile una periodica revisione alla luce dei risultati conseguiti nella ricerca scientifica.

Attraverso varie revisioni di questa classificazione internazionale, si arrivò nel 1948 alla $6^{a}$ edizione, che fu pubblicata per la prima volta a cura della WHO, fondata in quello stesso anno; tale classificazione venne adottata (e parzialmente) da soli 5 paesi. La sesta edizione dell'ICD incluse, per la prima volta, una sezione relativa ai disturbi mentali; in essa figuravano 10 categorie per le psicosi, 9 per le psiconevrosi e 7 per i disturbi del carattere, del comportamento e dell'intelligenza. Dopo altre due revisioni, nel 1977 apparve l'ICD-9 (WHO, 1977), che comprendeva in tutto 17 sezioni; tra esse la sezione $\mathrm{V}$ riguardava i disturbi mentali, ed includeva un totale di 30 categorie diagnostiche, con codici numerici a 3 cifre per la classificazione (001-999). Nell'ICD-9 non erano state elaborate delle definizioni operazionali dei disturbi, necessarie per far diagnosi con soddisfacente attendibilità, ma nelle varie categorie e sottocategorie era stato inserito un glossario con funzione di guida descrittiva delle varie sindromi.

Dopo soli cinque anni, ossia nel 1978, si avviò la progettazione della decima revisione del capitolo dell'ICD dedicato ai disturbi mentali. Così, dopo i primi anni dedicati ad un lavoro preparatorio, nel 1982 si tenne a Copenaghen una conferenza internazionale dedicata al problema della classificazione in psichiatria, alla quale presero parte oltre 150 psichiatri di 47 paesi; durante questo incontro furono definite le linee strategiche ispiratrici del futuro testo dell'ICD-10. Negli anni successivi sono stati pertanto preparati vari $d r a f t$, inviati a clinici ed esperti in tutto il mondo e quindi ampiamente rivisti alla luce dei commenti ricevuti da costoro. Inoltre, stretti contatti sono stati regolarmente mantenuti sia con le più importanti associazioni non governative del settore (ad esempio, la World Psychiatric Association, ed altre) che con associazioni nazionali, in particolare con la Task Force della American Psychiatric Association responsabile per la messa a punto del DSM-III, del DSM-III-R ed ora del DSM-IV; ciò al fine di migliorare l'omogeneità tra i più importanti sistemi diagnostici e di trarre mutuo vantaggio dall'intenso lavoro svolto da ciascun gruppo.

Il testo finale delle "Descrizioni Cliniche e Direttive Diagnostiche", da poco pubblicato (WHO, 1992), rappresenta pertanto il primo risultato di questo esteso sforzo collaborativo che ha coinvolto più 
di seicento esperti operanti in 52 paesi. Allorquando l'intero progetto relativo all'ICD-10 sarà concluso, esso comprenderà una intera famiglia di strumenti da utilizzare per molteplici fini e scopi in campo diagnostico-classificatorio; cosi, oltre al testo succitato, saranno disponibili i criteri diagnostici per la ricerca, un sistema classificatorio semplificato per l'uso nella medicina di base, degli adattamenti speciali dell'ICD-10 per settori determinati (ad esempio la psichiatria infantile) ed infine un manuale che consente di identificare le corrispondenze tra l'ICD-9, l'ICD-10 ed i principali sistemi di classificazione nazionale. La presenza di strumenti diversi, da impiegare in setting e per finalità molteplici, rappresenta una delle più significative differenze tra l'ICD-10 ed il DSM-III e IV (Frances et al., 1992). In questo contributo si fa riferimento esclusivamente al testo delle descrizioni cliniche e delle direttive diagnostiche, esistente nella sua forma ufficiale e finale: esso rappresenta una delle 22 sezioni in cui la decima revisione della classificazione internazionale delle malattie è suddivisa; tali sezioni coprono l'intera area della pratica medica.

Nell'ICD-10 è stato adottato un sistema di codificazione alfa-numerico basato su codici a tre elementi, dei quali il primo rappresentato da una lettera che indica il capitolo V e gli altri due, da 01 a 99, relativi alle maggiori categorie diagnostiche, a loro volta suddivise da una quarta cifra in specifiche sindromi o disturbi. Nel complesso, il capitolo V dell'ICD-10 comprende 100 categorie diagnostiche con codici a tre elementi, con un aumento quindi di tre volte rispetto al numero di categorie diagnostiche incluse nell'ICD-9. Non tutte queste categorie sono state per il momento utilizzate e questo of fre la possibilità di introdurre cambiamenti nella classificazione. La complessità del lavoro volto a predisporre un intero sistema classificatorio, e l'emergenza di nuove priorità, ha condotto infatti la WHO ad escludere una totale revisione dell'ICD-10 con periodicità decennale, come è accaduto sino ad oggi; l'attuale sistema, pertanto, rimarrà in vigore molto più a lungo, seppur con possibili parziali integrazioni e correzioni.

L'ICD-10 fornisce una descrizione introduttiva di ciascun disturbo, e comprende poi delle direttive diagnostiche che specificano il numero ed il tipo di sintomi che caratterizzano ciascuna sindrome o disturbo e che sono necessari per far diagnosi, mentre le indicazioni concernenti la durata dei disturbi sono da interpretare come un suggerimento di carattere generale piuttosto che come un criterio rigido. Anche per l'ICD-10 è prevista la messa a punto di un sistema multiassiale, che è tuttora in preparazione (Mezzich, 1988); allorquando sarà disponibile, esso conterrà cinque assi, non dissimili da quelli esistenti nel DSM-III e DSM-III-R. Va tuttavia notato che i disturbi di personalità sono stati classificati nell'asse principale insieme agli altri disturbi mentali ed alle sindromi comportamentali.

Per quanto riguarda altri punti da notare relativi all'ICD-10, si può ricordare che sia il termine psicogeno che psicosomatico non sono stati in genere impiegati nel testo a causa dei differenti significati che essi possono assumere nei vari linguaggi e nelle varie tradizioni psichiatriche. Inoltre, è apertamente sollecitata, ogni qualvolta la situazione lo richiede, la registrazione di più diagnosi, tante "quante sono necessarie per coprire l'intero quadro clinico"; in tali casi dovrebbe essere specificata quale è la diagnosi principale e quale la/e diagnosi secondaria/e; la diagnosi principale dovrebbe essere considerata quella relativa alla condizione morbosa all'origine del contatto con il servizio. È anche prevista la possibilità di formulare una diagnosi provvisoria (quando si attende di raccogliere ulteriori informazioni) o ipotetica (allorquando è improbabile che informazioni ulteriori divengano disponibili): in entrambi i casi è evidente che $\mathrm{i}$ criteri diagnostici sono soddisfatti soltanto in parte.

Per concludere, si deve sottolineare che;

"Queste descrizioni e direttive non hanno implicazioni teoriche, e non pretendono di rappresentare una messa a punto esauriente delle conoscenze attuali sulle varie condizioni morbose. Esse rappresentano semplicemente un insieme di sintomi e di osservazioni su cui è stato raggiunto un accordo da parte di un ampio numero di consulenti di vari paesi, e una base ragionevole per definire i limiti tra le categorie nella classificazione delle sindromi e dei disturbi psichici» (WHO, 1992).

\section{LE PRINCIPALI NOVITA DELL'ICD-10 RISPETTO ALL'ICD-9}

Per quanto riguarda le principali differenze tra l'ICD-10 e l'ICD-9, va innanzitutto notato che la suddivisione tra nevrosi e psicosi è stata abbandonata, mentre i due termini sono stati ancora occasionalmente impiegati nella denominazione di alcune specifiche sindromi (come ad esempio l'F40-48, Sindromi nevrotiche, legate a stress e somatoformi, o F23, Sindromi psicotiche acute e transitorie). Pertanto, invece di seguire la classica dicotomia nevrosi- 
psicosi, le sindromi ed i disturbi sono stati riuniti in specifici gruppi sulla base della loro affinità a livello clinico, il che è apparsa una soluzione praticamente più vantaggiosa.

Altre significative novità sono state introdotte nella classificazione delle sindromi attribuite ad una causa organica, che sono state tutte raggruppate nella sezione F00-F09. Per quanto attiene alla classificazione dei disturbi dovuti all'uso di sostanze psicoattive, il terzo elemento del codice viene impiegato per indicare la sostanza usata, mentre il quarto e quinto elemento si riferiscono alla sindrome psicopatologica in atto; in tal modo è possibile registrare tutte le condizioni morbose legate all'uso di sostanze.

L'ICD-10 ha incluso poi, per la prima volta, vari disturbi del comportamento nell'adulto, quali il gioco d'azzardo patologico, la piromania e la cleptomania. I disturbi della preferenza sessuale sono stati chiaramente differenziati dai disturbi dell'identità sessuale, e l'omosessualità di per sé non è stata più considerata quale categoria patologica, salvo che non sia accompagnata da altri disturbi o non sia egodistonica.

\section{FIELD TRIALS DELL'ICD-10}

Il testo provvisorio dell'ICD-10, messo a punto nel 1987, è stato utilizzato per i cosiddetti "field trials", nell'ambito di un vasto progetto di ricerca volto a valutare (1) se le direttive diagnostiche provviste per ciascun disturbo, o gruppo di disturbi, possono essere facilmente comprese ed impiegate da clinici in differenti paesi ed in differenti tipi di strutture socio-sanitarie; (2) se le varie categorie diagnostiche, e la descrizione fornita, sono congruenti con i casi visti nella comune pratica clinica nei differenti paesi; ed infine (3) se vari psichiatri possono raggiungere, dopo essersi familiarizzati con il testo delI'ICD-10, un soddisfacente livello di accordo diagnostico (Sartorius et al., 1993b). In questo modo in 112 centri, ubicati in 39 paesi, 711 clinici hanno condotto 15302 valutazioni diagnostiche; una fase di queste valutazioni prevedeva un esame congiunto di vari pazienti da parte di due o più clinici, dei quali uno effettuava l'intervista diagnostica mentre l'altro (o gli altri) assistevano, al fine di valutare il grado di accordo diagnostico tra essi. Ogni clinico poteva formulare una diagnosi principale, e sino a due diagnosi sussidiarie, quando ciò appariva necessario; pote- vano anche essere formulate una diagnosi alternativa alla diagnosi principale, e due diagnosi sussidiarie alternative. Ai clinici era quindi chiesto di valutare in che misura la diagnosi e la descrizione fornita dall'ICD-10 si adattavano al caso considerato (goodness of fit), ossia se tale descrizione includeva i principali sintomi riscontrabili sul piano clinico ed escludeva invece sintomi non pertinenti, ed infine se le diagnosi alternative erano improbabili. Veniva quindi anche chiesto di valutare se la diagnosi appariva facile o difficile da formulare utilizzando i criteri dell'ICD-10. Un totale di 568 clinici, in 97 centri in 35 paesi, hanno partecipato a questa fase dei field trials, portando a termine ben 9276 valutazioni di 2460 pazienti ambosessi, caratterizzati da un ampio range di età. Il numero medio di diagnosi fatte per paziente è stato di 1,74. La tabella II riporta i valori di $\mathrm{K}$ (che esprime il rapporto tra la concordanza osservata e la concordanza casuale tra osservatori diversi) per le 15 maggiori categorie diagnostiche a tre codici, per ciascuna delle quali furono valutati almeno 20 pazienti. Mentre per la maggior parte delle categorie considerate il livello di accordo diagnostico è apparso soddisfacente, per soli quattro gruppi di disturbi è emerso un basso livello di concordanza $(\mathrm{K}<.40)$ : (1) Altre sindromi psicotiche non organiche; (2) Stati affettivi persistenti; (3) Altri episodi affettivi e (4) Accentuazione dei tratti della personalità. Val la pena di notare che, proprio sulla base dei risultati emersi nei field trials, quest'ultima categoria diagnostica non è stata inclusa nella versione finale dell'ICD-10, mentre nel caso delle due precedenti il testo è stato adeguatamente rivisto. In totale, il valore di $\mathrm{K}$ per le 31 categorie a tre codici è stato pari a .71 , valore che può essere considerato largamente soddisfacente. Nella Tabella II sono anche riportate le percentuali dei clinici che ritenevano ottimo o buono il livello di congruenza tra la categoria diagnostica, la descrizione fornita ed il caso esaminato, e le percentuali di coloro che giudicavano i criteri diagnostici forniti molto facili o moderatamente facili da utilizzare: è agevole constatare le elevate percentuali di clinici che hanno espresso valutazioni in tal senso positive.

Tra le 55 categorie a quattro codici, 21 hanno riportato un valore di $\mathrm{K}$ più basso di .40 ; tra esse figuravano disturbi quali la sindrome affettiva organica, il disturbo di personalità organico, la schizofrenia catatonica, la sindrome psicotica acuta schizofrenosimile, la distimia, la sindrome mista ansioso-depressiva, e cinque disturbi di personalità. In generale si tratta di disturbi intorno ai quali è tradizionalmente 
Tabella II. - Coefficienti di Kappa, livello di congruenza delle diagnosi con i casi e facilità di impiego delle 15 principali categorie diagnostiche a tre codici dell'ICD-10 (versione del giugno 1987) nei «field trials».

\begin{tabular}{|c|c|c|c|c|}
\hline Codice e disturbo & pazienti (n) & Valore di kappa & $\begin{array}{l}\text { Livello di congruenza } \\
\text { con il caso ottimo } \\
\text { o buono }(\%)\end{array}$ & $\begin{array}{l}\text { Diagnosi molto facile o } \\
\text { moderatamente facile }(\%)\end{array}$ \\
\hline
\end{tabular}

\begin{tabular}{|c|c|c|c|c|}
\hline $\begin{array}{l}\text { F01 Demenza nella malattia } \\
\text { di Alzheimer }\end{array}$ & 67 & .81 & 90,7 & 92,3 \\
\hline $\begin{array}{l}\text { F10 Sindromi e disturbi psichici } \\
\text { e comportamentali dovuti all'uso } \\
\text { di alcoll }\end{array}$ & 141 & .82 & 87,1 & 90,0 \\
\hline F20 Schizofrenia & 490 & .81 & 89,8 & 92,3 \\
\hline $\begin{array}{l}\text { F21 Sindrcme schizotipica } \\
\text { F23 Sindromi psicotiche acute }\end{array}$ & 101 & .47 & 69,8 & 73,5 \\
\hline e transitorie & 146 & .65 & 71,0 & 75,1 \\
\hline F31 Episodio depressivo & 343 & .66 & 81,8 & 84,6 \\
\hline $\begin{array}{l}\text { F32 Sindrome affettiva bipolare } \\
\text { F33 Sindrome depressiva ricor- }\end{array}$ & 259 & .81 & 92,5 & 94,5 \\
\hline $\begin{array}{l}\text { rente } \\
\text { F34 Disturbi affettivi persistenti }\end{array}$ & 302 & .69 & 85,2 & 87,4 \\
\hline (es.: distimia) & 117 & .35 & 76,5 & 77,6 \\
\hline F36 Sindromi schizoaffettive & 148 & .48 & 75,7 & 80,9 \\
\hline F40 Sindromi fobiche & 49 & .63 & 83,5 & 84,5 \\
\hline $\begin{array}{l}\text { F41 Altre sindromi ansiose } \\
\text { F42 Sindrome ossessivo-compul- }\end{array}$ & 143 & .55 & 77,6 & 83,5 \\
\hline $\begin{array}{l}\text { siva } \\
\text { F43 Reazioni a gravi stress }\end{array}$ & 71 & .81 & 90,6 & 96,0 \\
\hline e sindromi da disadattamento & 140 & .60 & 74,3 & 81,6 \\
\hline F60 Disturbi della personalità & 211 & .47 & 67,9 & 72,3 \\
\hline
\end{tabular}

Dati tratti da Sartorius et al. (1993).

più arduo l'accordo diagnostico, e per i quali i criteri diagnostici sono stati rivisti al fine di migliorarne l'attendibilità e la precisione; tuttavia, i risultati complessivi emersi dai field trials sono tali da consentire di sostenere che l'ICD-10 ha incontrato un largo favore tra i numerosi clinici che hanno collaborato alla sua messa a punto.

\section{CONCLUSIONI}

"La classificazione è un modo di vedere il mondo" (Sartorius, 1991), e rappresenta un passaggio obbligato e necessario per rendere possibile la sistematizzazione delle conoscenze, per generare nuove ipotesi da mettere alla prova e per facilitare la comunicazione tra gli operatori. Nel contempo, per la specificità dei problemi con cui la psichiatria deve confrontarsi, qualsiasi sistema classificatorio, con la sua traduzione operativa (la diagnosi), incontra difficoltà sia sul piano teorico che operativo. La decima revisione dell'ICD rappresenta lo sforzo più ampio si- nora compiuto in campo psichiatrico per mettere a punto un sistema classificatorio e diagnostico utilizzabile da clinici e ricercatori diversi e che, nel contempo, sintetizzi approcci e culture psichiatriche differenziate. Il vero problema sarà ora quello di far sì che il testo venga davvero conosciuto dagli operatori ed impiegato nella pratica clinica. A tale proposito va ricordato che una ricerca americana, condotta di recente tra 658 psichiatri, ha dimostrato che erano pochissimi coloro che utilizzavano davvero i criteri del DSM-III nella propria pratica clinica, nonostante che la grande maggioranza dei clinici consultati fossero degli aperti sostenitori del sistema diagnostico in questione, e che tra essi vi fossero rappresentati anche alcuni ricercatori che avevano partecipato alla stesura del manuale diagnostico in questione: ad esempio, nessuno di essi affermò di impiegare tutti i criteri di inclusione ed esclusione per la diagnosi di depressione e mania, ed uno solo affermò di impiegare tutti i criteri previsti per la diagnosi di schizofrenia (Jampala et al., 1988). In un'altra ricerca effettuata nel 1982 tra il 5\% degli psichiatri iscritti all'American Psychiatric Association, ai quali fu chie- 
sto di specificare quali criteri essi di solito impiegassero per formulare una diagnosi di schizofrenia, solo una percentuale molto piccola (pari a circa il $5 \%$ dei clinici consultati) fece riferimento al DSM-III, e solo 2 psichiatri (su un totale di 341) fornirono dei criteri che erano simili a quelli propri del DSM-III (Lipkowitz \& Idupuganti, 1983, 1985).

Il problema vero, quindi, che si porrà nei prossimi anni sarà relativo alla reale diffusione ed assimilazione dell'ICD-10 tra i clinici: si ripropone quindi, ancora, il dilemma tra "acquisizione" ed "applicazione" delle conoscenze (de Girolamo, in stampa). Solo i prossimi anni potranno dire in che misura tale dilemma, nel caso dell'ICD-10, sarà stato risolto; questo contributo vuole essere un piccolo sforzo in questa direzione.

Ringraziamenti: Si ringraziano il Dr. Stylianos Nicolaou e la Dr.ssa Elisabetta Paitrinieri per aver collaborato alla stesura delle sezioni relative all'ICD-10; il Dr. Nicolaou ha anche collaborato alla preparazione finale del manoscritto. Si ringrazia in maniera particolare il Prof. Giovanni Federspil (Università di Padova) per aver fornito importanti ed accurati suggerimenti. Infine si ringrazia il Dr. Vincent Barras (Università di Ginevra) per aver fornito degli utili commenti.

\section{BIBLIOGRAFIA}

American Psychiatric Association (1980). Diagnostic and Statistical Manual of Mental Disorders (DSM III) (3rd ed.). American Psychiatric Association: Washington DC.

American Psychiatric Association (1987). Diagnostic and Statistical Manual of Mental Disorders (DSM III) (3rd ed. revised), American Psychiatric Association: Washington DC.

Antiseri D. (1983). Diagnosi clinica, logica della situazione e «occhio clinico". In Scoperta e Diagnosi in Medicina (ed. C. Scandellari e G. Federspil), pp. 97-128. Piccin: Padova.

Baldini M. (1975). Epistemologia Contemporanea e Clinica Medi$c a$. Città di Vita: Firenze.

Barras V. (1990). Semiologie et medicine. Gazette Medicale 18, 1869-1872.

Bentall R.P. (1992). A proposal to classify happiness as a psychiatric disorder. Journal of Medical Bioethics 18, 94-98.

Berlinguer G. (1982). Disease as suffering, deviation, danger, signal and stimulus. International Journal of Health Services 12, 309-319.

Blacker D. \& Tsuang M.T. (1992). Contested boundaries of bipolar disorder and the limits of categorical diagnosis in psychiatry. American Journal of Psychiatry 149, 1473-1483.

Blois M.S. (1988). Medicine and the nature of vertical reasoning. New England Journal of Medicine 318, 847-851.

Brown P. (1990). The name game: toward a sociology of diagnosis. In Challenging the Therapeutic State: Critical Perspectives on Psychiatry and the Mental Health System (ed. D. Cohen), Journal of Mind and Behaviour Special Issue, 11, 385-406.

Campbell E.J.M. (1987). The diagnosing mind. Lancet i, 849-851.
Cooper B. \& Morgan H.G. (1981). Psichiatria Epidemiologica. Boringhieri: Torino.

Cooper J.E. (1984). Diagnosi e processo diagnostico. In Manuale Cambridge di Psichiatria. 1. Psicopatologia Generale (ed. M. Shepherd e O.L. Zangwill), pp. 205-215. Zanichelli: Bologna.

Cooper J.E. (1985). La standardizzazione della valutazione e della classificazione in psichiatria. In L'Approccio Epidemiologico in Psichiatria (ed. M. Tanselia), pp. 50-63. Boringhieri: Torino.

Cooper J.E. (1988). The structure and presentation of contemporary psychiatric classifications with special reference to ICD-9 and 10. British Journal of Psychiatry Supplement No.1, 152, 21-28.

Cooper J.E., Kendell R.E., Gurland B.J., Sharpe L., Copeland J.R.M. \& Simon R. (1972). Psychiatric Diagnosis in New York and London: A Comparative Study of Mental-Hospital Admissions. (Maudsley Monograph No. 22). Oxford University: London.

de Girolamo G. (1991). Schizofrenia: dal mito kraepeliano ai modelli psicosociali. In Schizofrenia e guarigione, (ed. R. Warner), pp. 15-59. Feltrinelli: Milano.

de Girolamo G. (1993). International perspectives on the treatment and prevention of post-traumatic stress disorders. In International Handbook of Traumatic Stress Syndromes (ed. J.P. Wilson and B. Raphael), pp. 935-946. Plenum: New York.

de Girolamo G. (in stampa). Mental health research: ten unresolved dilemmas. Neurologia, Psichiatria e Scienze Umane.

de Girolamo G., Marchitelli G. \& Palazzi S. (1989). Sindrome premestruale: verso una nuova isteria? Rivista Sperimentale di Freniatria 113, 113-154.

Editorial (1987). Diagnosis: logic and psycho-logic. Lancet 1, 840841.

Eisenberg L. (1983). The subjective in medicine. Perspectives in Biology and Medicine 27, 48-61.

Fava G.A. \& Kellner R. (1993). Staging: a neglected dimension in psychiatric classification. Acta Psychiatrica Scandinavica 87, 225-230.

Federspil G. (1980). I Fondamenti del Metodo in Medicina Clinica e Sperimentale. Piccin: Padova.

Federspil G. (1982). La psichiatria come scienza: limiti e prospettive. In Controversie in Psichiatria (ed. V. Andreoli), pp. 57. 84. Masson: Milano.

Federspil G. (1990). La malattia come evento biologico. Minerva Medica 81, 845-854.

Federspil G. Nosografia. In Aggiornamenti della Enciclopedia Italiana. Istituto della Enciclopedia Italiana: Roma (in stampa).

Federspil G. \& Scandellari C. (1986). Dalla nosografia morgagnana alla nosografia contemporanea. In De Sedibus at Causis (eds. V. Cappelletti \& F. Di Trocchio), pp. 303-318. Istituto della Enciclopedia Italiana: Roma.

Feinstein A.R. (1972). Clinical biostatistics XIII: on homogeneity, taxonomy and nosography. Clinical Pharmacology and Therapeutics 13, 114-129.

Feinstein A.R. (1977). A critical overview of diagnosis in psychiatry. In Psychiatric Diagnosis (ed. V.M. Rakoff, H.C. Stancer and H.B. Kedward), pp. 189-206. Brunner Mazel: New York.

Feinstein A.R. (1985). Clinical Epidemiology: The Architecture of Clinical Research. W.B. Saunders Company: Philadelphia.

Foucault M. (1969). Nascita della Clinica. Einaudi: Torino.

Frances A., Pincus H., Widiger T., First M., Davis W. \& Hall W. (1992). DSM-IV and international communication in psychiatric diagnosis. World Psychiatry 1, 27-32. 
Gauron E.F. \& Dickinson J.K. (1966). Diagnostic decision making in psychiatry. Archives of General Psychiatry 14, 225237.

Gauron, E.F. \& Dickinson J.K. (1969). The influence of seeing the patient first on diagnostic decision making in psychiatry. American Journal of Psychiatry 126, 199-205.

Goldberg D. \& Huxley P. (1992). Common Mental Disorders. A Bio-social Model. Tavistock: London. Trad. it.: Disturbi Emotivi Comuni. Un Approccio Biosociale (ed. M. Tansella). Il Pensiero Scientifico: Roma, 1993

Hempel C.G. (1968). Filosofia delle Scienze Naturali. Il Mulino: Bologna.

Grove W.M., Andreasen N.C., McDonald-Scott P., Keller M.B. \& Shapiro R.W. (1981). Reliability studies of psychiatric diagnosis. Archives of General Psychiatry 38, 408-413.

Hollister L.E. (1992). Panic disorder: old wine in new bottles. Integrative Psychiatry 8, 75-83, 1992.

Jablensky A. (1988). Methodological issues in psychiatric classification. British Journal of Psychiatry Supplement No.1, 152, 15-20.

Jampala C.V., Sierles F.S. \& Taylor M.A. (1986). Consumers views of DSM-III: attitudes and practices of US psychiatrists and 1984 graduating psychiatric residents. American Journal of Psychiatry 143, 148-153.

Jampala V., Sierles F.S. \& Taylor M.A. (1988). The use of DSMIII in the United States: a case of not going by the book. Comprehensive Psychiatry 29, 39-47.

Jaspers K. (1964). Psicopatologia Generale. Il Pensiero Scientifico: Roma.

Kendell R.E. (1974). Il Ruolo della Diagnosi in Psichiatria. Il Pensiero Scientifico: Roma.

Kendell R.E. (1984). I principi classificatori in relazione alla malattia mentale. In Manuale Cambridge di Psichiatria. 1. Psicopatologia Generale (ed. M. Shepherd e O.L. Zangwill), pp. 197-204. Zanichelli: Bologna.

Kendell R.E. (1989). Clinical validity. In The Validity of Psychiatric Diagnosis (ed. L.N. Robins and J.E. Barret), pp.305-323. Raven Press: New York.

Kendell R.E. (1991). Relationship between the DSM-IV and the ICD-10. Journal of Abnormal Psychology 3, 297-301.

Lanteri-Laura G. (1983). La semiologie psychiatrique: son evolution et son etat en 1982. L'Evolution Psichiatrique 48, 327363.

Lanteri-Laura G. \& Del Pistoia L. (1983). Il sapere psichiatrico e le condizioni della sua produzione. Rivista Sperimentale di Freniatria 107, 34-49.

Lazare A. (1973). Hidden conceptual models in clinical psychiatry. New England Journal of Medicine 288, 345-351.

Lipkowitz M.H. \& Idupuganti S. (1983). Diagnosing schizophrenia in 1982: the effect of DSM-III. American Journal of Psychiatry 142, 634-637.

Lipkowitz M.H. \& Idupuganti S. (1985). Diagnosing schizophrenia in 1980: a survey of US physicians. American Journal of Psychiatry 140, 52-55.

Lipton A.A. \& Simon F.S. (1985). Psychiatric diagnosis in a state hospital: Manhattan State revisited. Hospital and Community Psychiatry 36, 368-373.

Llyod G.G. (1986). Hysteria: a case for conservation? British Medical Journal 293, 1255-1256.

Loranger A.W. (1990). The impact of DSM-III on diagnostic practice in a University Hospital. Archives of General Psychiatry 47, 672-675.

Loranger A.W., Hirschfeld R.M.A., Sartorius N. \& Regier D.A.
(1991). The WHO/ADAMHA International Pilot Study of Personality Disorders: background and purpose. Journal of Personality Disorders 5, 296-306.

Loring M. \& Powell B. (1988). Gender, race, and DSM-III: a study of the objectivity of psychiatric diagnostic behaviour. Journal of Health and Social Behavior 29, 1-22.

Meehl P.E. (1985). Diagnostic taxa as open concepts: metatheoretical and statistical questions about reliability and construct validity in the grand strategy of nosological revision. In Contemporary Directions in Psychopathology: Toward the DSM$I V$ (ed. T. Millon and G.L. Klerman), pp. 215-231. Guilford: New York.

Mezzich J.E. (1988). On developing a psychiatric multiaxial schema for ICD-10. British Journal of Psychiatry, Supplement No.1, 152, 38-43.

Millon T. (1991). Classification in psychopathology: rationale, alternatives, and standards. Journal of Abnormal Psychology 3, 245-261.

Parshall A.M. \& Priest R.G. (1993). Nosology, taxonomy and the classification conundrum of the functional psychosis. British Journal of Psychiatry 162, 227-236.

Pera M. (1982). Metodologie della scienza e filosofie della mente. La psichiatria al bivio. In Controversie in Psichiatria (ed. V. Andreoli), pp. 85-98. Masson: Milano.

Ponsi I. \& Bonner M. (1979). Modelli di malattia mentale e di intervento psichiatrico. Psicoterapia e Scienze Umane 3, 47-68.

Pull C.B. \& Wittchen H.U. (1991). CIDI, SCAN and IPDE: structured diagnostic interviews for ICD-10 and DSM-III-R. European Psychiatry 6, 277-285.

Robins L.N., Wing J,, Wittchen H.-U., Helzer J.E., Babor T.F., Burke J., Farmer A., Jablenski A., Pickens R., Regier D.A., Sartorius N. \& Towle L.H. (1988). The Composite International Diagnostic Interview. Archives of General Psychiatry 45, 1069-1076.

Robins L.N. (1990). Psychiatric epidemiology - a historic review. Social Psychiatry and Psychiatric Epidemiology 25, 16-26.

Rosaia L. (1986). L'incertezza della medicina. Le Scienze 209, 3239.

Rutter M. \& Gould M. (1985). Classification. In Child and Adolescent Psychiatry. Modern Approaches. (ed. M. Rutter and L. Hersov), pp. 304-321. Blackwell: London.

Sandifer M.G., Hordern A. \& Green L.M. (1970). The psychiatric interview: the impact of the first three minutes. American Journal of Psychiatry 126, 968-973.

Sartorius N. (1991). The classification of mental disorders in the Tenth Revision of the International Classification of Diseases. European Psychiatry 6, 315-322.

Sartorius N., de Girolamo G., Andrews G., German A. \& Eisenberg L. (eds.) (1993a). Treatment of Mental Disorders. A Review of Effectiveness. American Psychiatric Press: Washington.

Sartorius N., Kaelber C., Cooper J., Roper M., Rae D., Gulbinat W., Ustun B. \& Regier D. (1993b). Progress towards achieving a common language in psychiatry: results from the field trial of the clinical guidelines accompanying the WHO classification of mental and behavioural disorders in the ICD-10. Archives of General Psychiatry 50, 115-124..

Scadding J.G. (1988). Health and disease: whan can medicine do for philosophy? Journal of Medical Bioethics 14, 118-124.

Scadding G. (1993). Nosology, taxonomy and the classification conundrum of the functional psychoses. British Journal of Psychiatry 162, 237-238.

Scandellari C. (1981). La Strategia della Diagnosi. Piccin: Padova. 
Scandellari C. \& Federspil G. (ed.) (1983). Scoperta e Diagnosi in Medicina. Piccin: Padova.

Shepherd M., Brooke E.M., Cooper J.E. \& Lin T. (1968). An Experimental Approach to Psychiatric Diagnosis. Acta Psychiatrica Scandinavica, Supplementum No. 201, vol. 44, pp. 7-86.

Sokal R.R. (1974). Classification: purposes, principles, progress. Science 185, 115-123.

Spitzer R.L., Endicott J. \& Robins E. (1978). Reliability of clinical criteria for psychiatric diagnosis. In Psychiatric Diagnosis: Exploration of Biological Predictors (ed. H.S. Akiskal and W.L. Webb), pp. 61-73. SP Medical \& Scientific Books: New York.

Szasz T. (1991). Diagnoses are not diseases. Lancet 338, 15741576.

Temerlin M.K. (1968). Suggestion effects in psychiatric diagnosis. Journal of Nervous and Mental Disease 147, 349-353.

Terzian E. (1993). Panico: una revisione (non esaustiva) della letteratura. Lettera (Istituto Mario Negri), 132-181

Wallace E.R. (1988). What is "Truth»? some philosophical con- tributions to psychiatric issues. American Journal of Psychiatry 145, 137-147.

Williams, P. (1979) Deciding how to treat - the relevance of psychiatric diagnosis. Psychological Medicine, 9 179-186.

Wing J.K., Babor T., Brugha T., Burke J., Cooper J.E., Giel R., Jablenski A., Regier D. \& Sartorius N. (1990). SCAN: Schedules for clinical assessment in neuropsychiatry. Archives of General Psychiatry 47, 589-593.

World Health Organization (1977). International Classification of Diseases, 9th Revision. WHO: Geneva.

World Health Organization (1979). The International Pilot Study of Schizophrenia. A Follow-up Study. Wiley: Chichester.

World Health Organization (1992). International Classification of Diseases, 10th. Revision. WHO: Geneva. Ed. it.: Decima Revisione della Classificazione Internazionale delle Sindromi e dei Disturbi Psichici e Comportamentali. Descrizioni Cliniche e Direttive Diagnostiche (ed. D. Kemali, M. Maj, F. Catapano, S. Lobrace e L. Magliano). Masson: Milano. 


\section{A. Scott Henderson}

\section{Psichiatria sociale ed epidemiologia psichiatrica}

Edizione italiana a cura di Michele Tansella

Un libro di 226 pagine. Lire 40.000

"L'opera di Scott Henderson rappresenta innanzitutto una fonte preziosa di informazioni e di dati, essendo l'autore riuscito a concentrare in nove capitoli i risultati dei principali studi di epidemiologia psichiatrica pubblicati finora.

Ma questo volume ha anche un altro pregio. Esso ricorda continuamente al lettore l'importanza dell'approccio epidemiologico e di un'adeguata metodologia di ricerca per testare in modo corretto le nostre ipotesi".

Dalla Presentazione del Curatore dell'edizione italiana

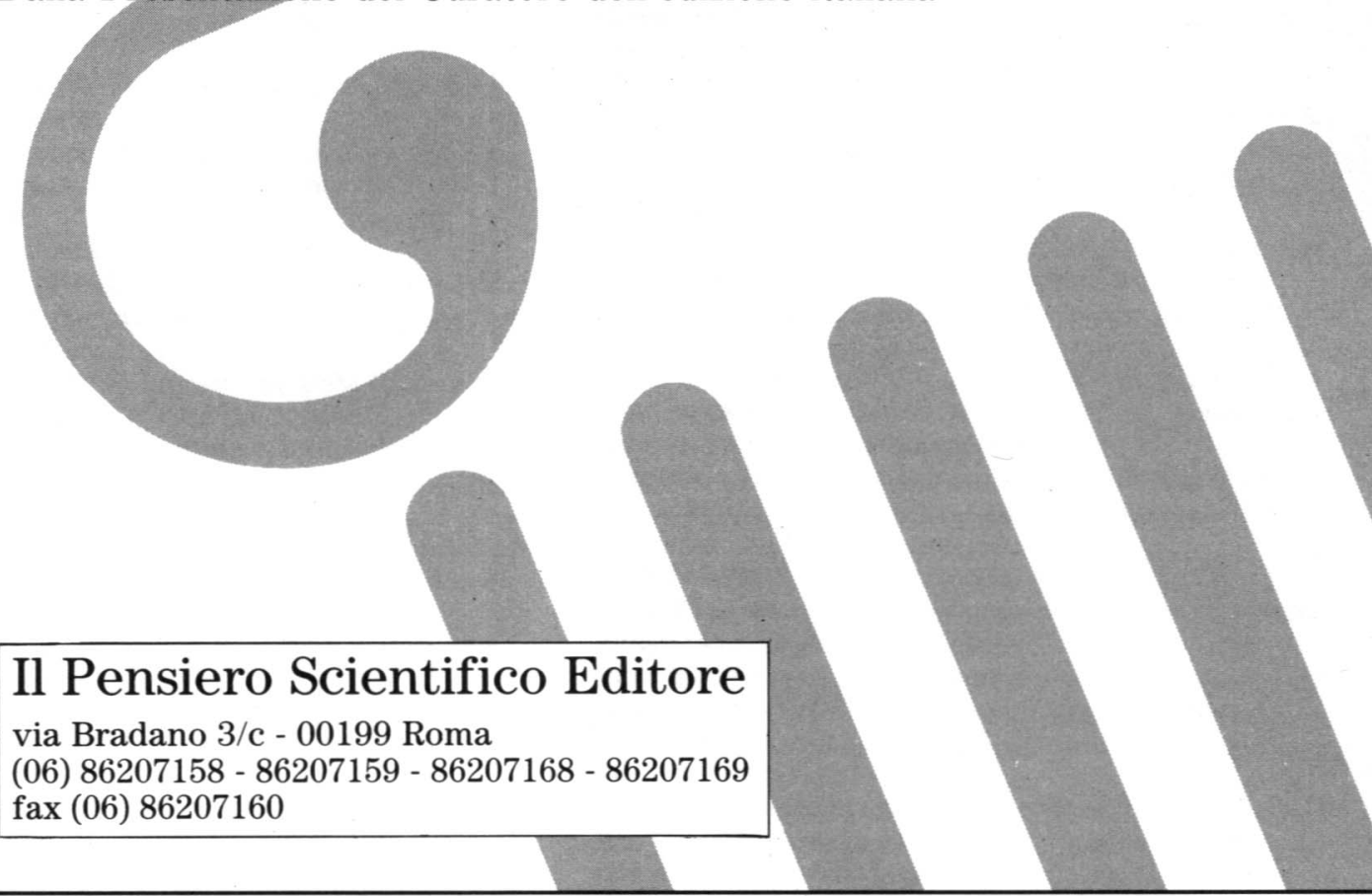

Compilate e spedite in busta chiusa a:

Il Pensiero Scientifico Editore

$\square$ SÌ DESIDERO RICEVERE N ${ }^{\circ}$ di "PSICHIATRIA SOCIALE"

al prezzo di Lire 40.000 la copia

VERSERÒ LA SOMMA DI LIRE

$\square$ VERSAMENTO C/C POSTALE N 902015
$\square$ VISA
$\square$ CARTASI
$\square$ MASTERCARD

$\square$ N.L. L

Cognome Nome

Indirizzo

Città

CAP Prov.

Tel.

Firma 